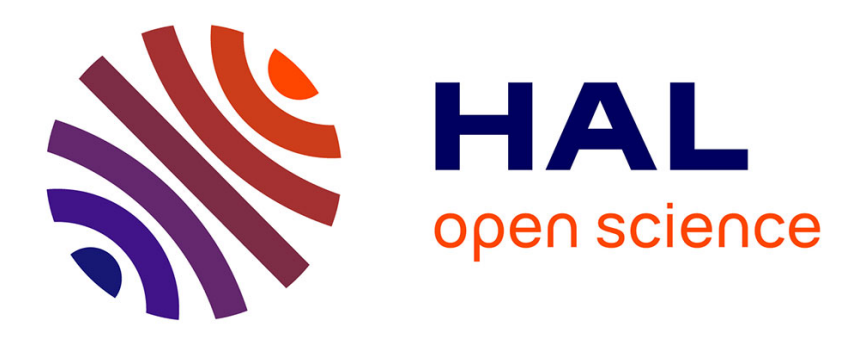

\title{
A Model of Optimal Portfolio Selection under Liquidity Risk and Price Impact
}

Huyen Pham, Vathana Ly Vath, Mohamed Mnif

\section{To cite this version:}

Huyen Pham, Vathana Ly Vath, Mohamed Mnif. A Model of Optimal Portfolio Selection under Liquidity Risk and Price Impact. 2005. hal-00011190

\author{
HAL Id: hal-00011190 \\ https://hal.science/hal-00011190
}

Preprint submitted on 12 Oct 2005

HAL is a multi-disciplinary open access archive for the deposit and dissemination of scientific research documents, whether they are published or not. The documents may come from teaching and research institutions in France or abroad, or from public or private research centers.
L'archive ouverte pluridisciplinaire HAL, est destinée au dépôt et à la diffusion de documents scientifiques de niveau recherche, publiés ou non, émanant des établissements d'enseignement et de recherche français ou étrangers, des laboratoires publics ou privés. 


\title{
A Model of Optimal Portfolio Selection under Liquidity Risk and Price Impact*
}

\author{
Vathana LY VATH $\quad$ Mohamed MNIF $\quad$ Huyên PHAM $^{\ddagger}$
}

October 12, 2005

\begin{abstract}
We study a financial model with one risk-free and one risky asset subject to liquidity risk and price impact. In this market, an investor may transfer funds between the two assets at any discrete time. Each purchase or sale policy decision affects the price of the risky asset and incurs some fixed transaction cost. The objective is to maximize the expected utility from terminal liquidation value over a finite horizon and subject to a solvency constraint. This is formulated as an impulse control problem under state constraint and we prove that the value function is characterized as the unique constrained viscosity solution to the associated quasi-variational Hamilton-Jacobi-Bellman inequality.
\end{abstract}

Key words : portfolio selection, liquidity risk, impulse control, state constraint, discontinuous viscosity solutions.

MSC Classification (2000) : 93E20, 91B28, 60H30, 49L25.

\footnotetext{
${ }^{*}$ We would like to thank Mihail Zervos for useful discussions.

${ }^{\dagger}$ Laboratoire de Probabilités et Modèles Aléatoires, CNRS, UMR 7599, Université Paris 7, France, lyvath@math.jussieu.fr

${ }^{\ddagger}$ LEGI, Ecole Polytechnique de Tunisie, mohamed.mnif@enit.rnu.tn

${ }^{\S}$ Laboratoire de Probabilités et Modèles Aléatoires, CNRS, UMR 7599, Université Paris 7, France, pham@math.jussieu.fr and CREST
} 


\section{Introduction}

Classical market models in mathematical finance assume perfect elasticity of traded assets : traders act as price takers, so that they buy and sell with arbitrary size without changing the price. However, the market microstructure literature has shown both theoretically and empirically that large trades move the price of the underlying assets. Moreover, in practice, investors face trading strategies constraints, typically of finite variation, and they cannot rebalance them continuously. We then usually speak about liquidity risk or illiquid markets. While the assumption of perfect liquidity market may not be practically important over a very long term horizon, price impact can have a significant difference over a short time horizon.

Several suggestions have been proposed to formalize the liquidity risk. In [22] and [2], the impact of trading strategies on prices is explained by the presence of an insider. In the market manipulation literature, prices are assumed to depend directly on the trading strategies. For instance, the paper [11] considers a diffusion model for the price dynamics with coefficients depending on the large investor's strategy, while [14], [25], [5] or [7] develop a continuous-time model where prices depend on strategies via a reaction function. We mention also in this direction a recent paper [8] in a discrete-time model. Notice that liquidity risk is also related to transaction cost literature, see e.g. [12] and [20], since transactions costs incur finite variation on trading strategies and bid/ask spead on the assets price. Most of these cited papers focus on the implications of their liquidity risk modelization on arbitrage and asset pricing.

In this paper, we propose a model of liquidity risk and price impact that adopts both perspectives of market manipulation and transaction cost literature. Our model is inspired from the recent papers [27] and [16], and may be described roughly as follows. Trading on illiquid assets is not allowed continuously due to some fixed costs but only at any discrete times. These liquidity constraints on strategies are in accordance with practitioner literature. There is an investor, who is large in the sense that his strategies affect asset prices : prices are pushed up when buying stock shares and moved down when selling shares. In this context, we study an optimal portfolio choice problem over a finite horizon : the investor maximizes his expected utility from terminal liquidation wealth and under a natural economic solvency constraint.

Our optimization problem is formulated as a parabolic impulse control problem with three variables (besides time variable) related to the cash holdings, number of stock shares and price. This problem is known to be associated by the dynamic programming principle to a Hamilton-Jacobi-Bellman (HJB) quasi-variational inequality, see [4]. We refer to [19], [21], [6] or [24] for some recent papers involving applications of impulse controls in finance, mostly over an infinite horizon and in dimension 1, except [21] and [24] in dimension 2. Moreover, in our context, the economic solvency condition requires that liquidation wealth is nonnegative, which is translated into a state constraint.

The features of our stochastic control problem make appear several technical difficulties related to the nonlinearity of the impulse transaction function and the solvency constraint. In particular, the liquidation net wealth may grow after transaction, which makes nontrivial 
the finiteness of the value function. Hence, the Merton bound does not provide as e.g. in transaction cost models, a natural upper bound on the value function. Instead, we provide a suitable "linearization" of the liquidation value that provides a sharp upper bound of the value function. The solvency region (or state domain) is not convex and its boundary even not smooth, in contrast with transaction cost model (see [12]), so that continuity of the value function is not direct. Moreover, the boundary of the solvency region is not absorbing as in transaction cost models and singular control problems, and the value function may be discontinuous on some parts of the boundary. In our general set-up, it is then natural to consider the HJB equation with the concept of (discontinuous) viscosity solutions, which provides by now a well established method for dealing with stochastic control problems, see e.g. the book [13]. More precisely, we need to consider constrained viscosity solutions to handle the state constraints. Our first main result is to prove that the value function is a constrained viscosity solution to its associated HJB quasi-variational inequality, by adapting arguments from [24]. Our second main result is a comparison principle for the state constraint HJB quasi-variational inequality, which ensures a PDE characterization for the value function of our problem. Previous comparison results derived for variational inequality (see [17], [28]) associated to impulse problem do not apply here. In our context, we prove that one can compare a subsolution with a supersolution to the HJB quasivariational inequality provided that one can compare them at the terminal date (as usual in parabolic problems) but also on some part $D_{0}$ of the solvency boundary. We follow general viscosity solutions technique, see e.g. [10], with specificities coming from the nonlocal impulse operator and the boundary conditions. To handle the impulse obstacle, and in the spirit of [18], we produce a suitable strict supersolution of the HJB quasi-variational inequality. For the boundary conditions and following [26], the general idea is to build a test function so that the minimum associated with the (strict) supersolution cannot be on the boundary. However, the method in [26] does not apply here since it requires the continuity of the supersolution on the boundary, which is precisely not the case in our model. Instead, we adapt a method in [3], which requires the smoothness of the boundary. This is the case here except on the part $D_{0}$ of the boundary, but for which one can prove directly the continuity of the value function.

The paper is organized as follows. In the next Section 2, we formulate our impulse control problem with solvency constraint. Section 3 recalls the HJB quasi-variational inequality associated to the problem, and states the main result. In Section 4, we derive several useful properties on the value function. Section 5 is devoted to the PDE viscosity characterization of the value function.

\section{Problem formulation}

Let $(\Omega, \mathcal{F}, \mathbb{P})$ be a probability space equipped with a filtration $\left(\mathcal{F}_{t}\right)_{0 \leq t \leq T}$ supporting an one-dimensional Brownian motion $W$ on a finite horizon $[0, T], T<\infty$. We consider a financial market consisting of a money market account yielding a constant interest rate $r$ $\geq 0$ and a risky asset (or stock) of price process $P=\left(P_{t}\right)$. We denote by $X_{t}$ the amount of money (or cash holdings) and by $Y_{t}$ the number of shares in the stock held by the investor 
at time $t$.

Liquidity constraints. We assume that the investor can only trade discretely on $[0, T)$. This is modelled through an impulse control strategy $\alpha=\left(\tau_{n}, \zeta_{n}\right)_{n \geq 1}: \tau_{1} \leq \ldots \tau_{n} \leq \ldots<$ $T$ are stopping times representing the intervention times of the investor and $\zeta_{n}, n \geq 1$, are $\mathcal{F}_{\tau_{n}}$-measurable random variables valued in $\mathbb{R}$ and giving the number of stock purchased if $\zeta_{n} \geq 0$ or selled if $\zeta_{n}<0$ at these times. The sequence $\left(\tau_{n}, \zeta_{n}\right)$ may be a priori finite or infinite. The dynamics of $Y$ is then given by :

$$
\begin{aligned}
Y_{s} & =Y_{\tau_{n}}, \quad \tau_{n} \leq s<\tau_{n+1} \\
Y_{\tau_{n+1}} & =Y_{\tau_{n}}+\zeta_{n+1} .
\end{aligned}
$$

Notice that we do not allow trade at the terminal date $T$, which is the liquidation date.

Price impact. The large investor affects the price of the risky stock $P$ by his purchases and sales : the stock price goes up when the trader buys and goes down when he sells and the impact is increasing with the size of the order. We then introduce a price impact positive function $Q(y, p)$ which indicates the post-order price when the large investor trades a position of $y$ shares of stock at a pre-order price $p$. In absence of price impact, we have $Q(y, p)=p$. Here, we have $Q(0, p)=p$ meaning that no trading incurs no impact and $Q$ is nondecreasing in $y$ with $Q(y, p) \geq($ resp. $\leq) p$ for $y \geq($ resp. $\leq)$ ) . Actually, in the rest of the paper, we consider a price impact function in the form

$$
Q(y, p)=p e^{\lambda y}, \quad \text { where } \lambda>0 .
$$

We denote $\theta(y, p)$ the transaction cost function which indicates the (algebraic) cost for a (large) investor with a position of $y$ shares in stock when the pre-trade price is $p$ :

$$
\theta(y, p)=y Q(y, p)
$$

The cost function $l \geq 0 \mapsto l Q(l, p)$ indicates the amount of money to be paid by the large investor for buying $l$ shares of stock when the pre-order price is $p$. The revenue function $m \geq 0 \mapsto m Q(-m, p)$ represents the amount of money obtained by the large investor for selling $m$ shares of stock when the pre-order price is $p$. The liquidation function $\ell(y, p)$ which represents the value that an investor would obtained by liquidating immediately his stock position by a single block trade is given by

$$
\ell(y, p)=-\theta(-y, p) .
$$

We then model the dynamics of the price impact as follows. In the absence of trading, the price process is governed by

$$
d P_{s}=P_{s}\left(b d s+\sigma d W_{s}\right)
$$

where $b, \sigma$ are constants with $\sigma>0$. When a discrete trading $\Delta Y_{s}:=Y_{s}-Y_{s^{-}}$occurs at time $s$, the price impact is

$$
P_{s}=Q\left(\Delta Y_{s}, P_{s^{-}}\right), \quad \text { i.e. } \quad \Delta P_{s}:=P_{s}-P_{s^{-}}=Q\left(\Delta Y_{s}, P_{s^{-}}\right)-P_{s^{-}}
$$


In other words, given a trading strategy $\left(\tau_{n}, \zeta_{n}\right)_{n \geq 1}$, the price process $P$ is given by

$$
\begin{aligned}
P_{s} & =P_{\tau_{n}} \frac{P_{s}^{0}}{P_{\tau_{n}}^{0}}, \quad \tau_{n} \leq s<\tau_{n+1}, \\
P_{\tau_{n+1}} & =Q\left(\zeta_{n+1}, P_{\tau_{n+1}^{-}}\right),
\end{aligned}
$$

where

$$
P_{s}^{0}=\exp \left(\left(b-\frac{\sigma^{2}}{2}\right) s+\sigma W_{s}\right)
$$

Notice that with this modelling of price impact, the price process $P$ is always strictly positive, i.e. valued in $\mathbb{R}_{+}^{*}=(0, \infty)$.

Cash holdings. In absence of transactions, the process $X$ grows deterministically at exponential rate $r$ :

$$
d X_{s}=r X_{s} d s
$$

When a discrete trading $\Delta Y_{s}$ occurs at time $s$ with pretrade price $P_{s^{-}}$, we assume that in addition to the transaction of stocks $\theta\left(\Delta Y_{s}, P_{s^{-}}\right)$, there is a fixed cost $k>0$ to be paid. This results in a variation of cash holdings by :

$$
\Delta X_{s}:=X_{s}-X_{s^{-}}=-\theta\left(\Delta Y_{s}, P_{s^{-}}\right)-k .
$$

In other words, the cash holdings process $X$ of the large investor is given by :

$$
\begin{aligned}
X_{s} & =X_{\tau_{n}} \frac{X_{s}^{0}}{X_{\tau_{n}}^{0}}, \quad \tau_{n} \leq s<\tau_{n+1}, \\
X_{\tau_{n+1}} & =X_{\tau_{n+1}^{-}}-\theta\left(\zeta_{n+1}, P_{\tau_{n+1}^{-}}\right)-k,
\end{aligned}
$$

with

$$
X_{s}^{0}=\exp (r s)
$$

The assumption that any trading incurs a fixed cost of money to be paid will rule out continuous trading, i.e. optimally, the sequence $\left(\tau_{n}, \zeta_{n}\right)$ is not degenerate in the sense that for all $n, \tau_{n}<\tau_{n+1}$ and $\zeta_{n} \neq 0$ a.s.

Liquidation value and solvency constraint. If the agent has the amount $x$ in the bank account, the number of shares $y$ of stocks at the preorder price $p$, i.e. a state value $z=$ $(x, y, p)$, his net wealth or liquidation value is given by :

$$
L(z)=\max \left[L_{0}(z), L_{1}(z)\right] 1_{y \geq 0}+L_{0}(z) 1_{y<0},
$$

where

$$
L_{0}(z)=x+\ell(y, p)-k, \quad L_{1}(z)=x .
$$

The interpretation is the following. $L_{0}(z)$ corresponds to the net wealth of the agent when he liquidates his position in stock. Moreover, if he has a long position in stock, i.e. $y \geq 0$, 
he can bin his stock shares, by keeping only his cash amount, which leads to a net wealth $L_{1}(z)$. This last possibility may be advantageous, i.e. $L_{1}(z) \geq L_{0}(z)$, due to the fixed cost $k$. Hence, globally, his net wealth is given by (2.4).

We then naturally introduce the liquidation solvency region (see Figure 1) :

$$
\mathcal{S}=\left\{z=(x, y, p) \in \mathbb{R} \times \mathbb{R} \times \mathbb{R}_{+}^{*}: L(z)>0\right\},
$$

and we denote its boundary and its closure by

$$
\partial \mathcal{S}=\left\{z=(x, y, p) \in \mathbb{R} \times \mathbb{R} \times \mathbb{R}_{+}^{*}: L(z)=0\right\} \quad \text { and } \quad \overline{\mathcal{S}}=\mathcal{S} \cup \partial \mathcal{S} .
$$

Remark 2.1 In the absence of liquidity risk, i.e $\lambda=0$, and fixed transaction cost, i.e. $k$ $=0$, we have the usual net wealth constraint : $L(z)=x+p y \geq 0$. In our context, the function $L$ is clearly continuous on $\left\{z=(x, y, p) \in \mathbb{R} \times \mathbb{R} \times \mathbb{R}_{+}^{*}: y \neq 0\right\}$. It is discontinuous on $z_{0}=(x, 0, p) \in \overline{\mathcal{S}}$, but it is easy to check that it is upper-semicontinuous on $z_{0}$, so that globally $L$ is upper-semicontinuous. Hence $\overline{\mathcal{S}}$ is closed in $\mathbb{R} \times \mathbb{R} \times \mathbb{R}_{+}^{*}$.

Remark 2.2 For any $p>0$, the function $y \mapsto \ell(y, p)=p y e^{-\lambda y}$ is increasing on $[0,1 / \lambda]$, decreasing on $[1 / \lambda, \infty)$ with $l(0, p)=\lim _{y \rightarrow \infty} l(y, p)=0$ and $l(1 / \lambda, p)=p e^{-1} / \lambda$. We then distinguish the two cases :

$\star$ if $p<k \lambda e$, then $l(y, p)<k$ for all $y \geq 0$.

$\star$ if $p \geq k \lambda e$, then there exists an unique $y_{1}(p) \in(0,1 / \lambda]$ and $y_{2}(p) \in[1 / \lambda, \infty)$ such that $l\left(y_{1}(p), p\right)=l\left(y_{2}(p), p\right)=k$ with $l(y, p)<k$ for all $y \in\left[0, y_{1}(p)\right) \cup\left(y_{2}(p), \infty\right)$. Moreover, $y_{1}(p)$ (resp. $y_{2}(p)$ ) decreases to 0 (resp. increases to $\infty$ ) when $p$ goes to infinity, while $y_{1}(p)$ (resp. $\left.y_{2}(p)\right)$ increases (resp. decreases) to $1 / \lambda$ when $p$ decreases to $k \lambda e$.

The boundary of the solvency region may then be explicited as follows (see Figures 2 and 3) :

$$
\partial \mathcal{S}=\partial_{\ell}^{-} \mathcal{S} \cup \partial^{y} \mathcal{S} \cup \partial_{0}^{x} \mathcal{S} \cup \partial_{1}^{x} \mathcal{S} \cup \partial_{2}^{x} \mathcal{S} \cup \partial_{\ell}^{+} \mathcal{S}
$$

where

$$
\begin{aligned}
& \partial_{\ell}^{-} \mathcal{S}=\left\{z=(x, y, p) \in \mathbb{R} \times \mathbb{R} \times \mathbb{R}_{+}^{*}: x+\ell(y, p)=k, y \leq 0\right\} \\
& \partial^{y} \mathcal{S}=\left\{z=(x, y, p) \in \mathbb{R} \times \mathbb{R} \times \mathbb{R}_{+}^{*}: 0 \leq x<k, y=0\right\} \\
& \partial_{0}^{x} \mathcal{S}=\left\{z=(x, y, p) \in \mathbb{R} \times \mathbb{R} \times \mathbb{R}_{+}^{*}: x=0, y>0, p<k \lambda e\right\} \\
&\left.\partial_{1}^{x} \mathcal{S}=\left\{z=(x, y, p) \in \mathbb{R} \times \mathbb{R} \times \mathbb{R}_{+}^{*}: x=0,0<y<y_{1}(p)\right), p \geq k \lambda e\right\} \\
& \partial_{2}^{x} \mathcal{S}=\left\{z=(x, y, p) \in \mathbb{R} \times \mathbb{R} \times \mathbb{R}_{+}^{*}: x=0, y>y_{2}(p), p \geq k \lambda e\right\} \\
& \partial_{\ell}^{+} \mathcal{S}=\left\{z=(x, y, p) \in \mathbb{R} \times \mathbb{R} \times \mathbb{R}_{+}^{*}: x+\ell(y, p)=k, y_{1}(p) \leq y \leq y_{2}(p), p \geq k \lambda e\right\} .
\end{aligned}
$$

In the sequel, we also introduce the corner lines in $\partial \mathcal{S}$ :

$$
\begin{aligned}
D_{0} & =\{(0,0)\} \times \mathbb{R}_{+}^{*} \subset \partial^{y} \mathcal{S} \\
D_{k} & =\{(k, 0)\} \times \mathbb{R}_{+}^{*} \subset \partial_{\ell}^{-} \mathcal{S} \\
C_{1} & =\left\{\left(0, y_{1}(p), p\right): p \in \mathbb{R}_{+}^{*}\right\} \subset \partial_{\ell}^{+} \mathcal{S} \\
C_{2} & =\left\{\left(0, y_{2}(p), p\right): p \in \mathbb{R}_{+}^{*}\right\} \subset \partial_{\ell}^{+} \mathcal{S} .
\end{aligned}
$$




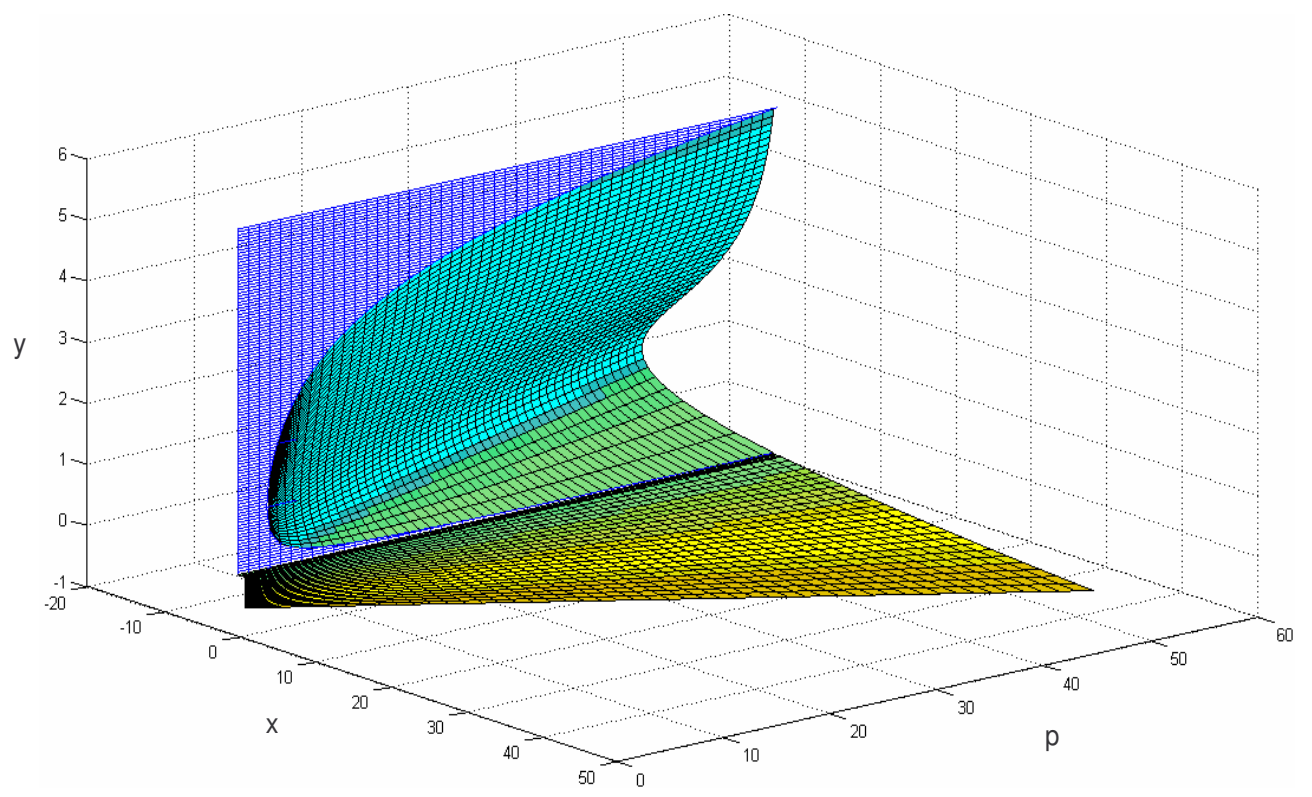

Figure 1: The solvency region when $k=1, \lambda=1$

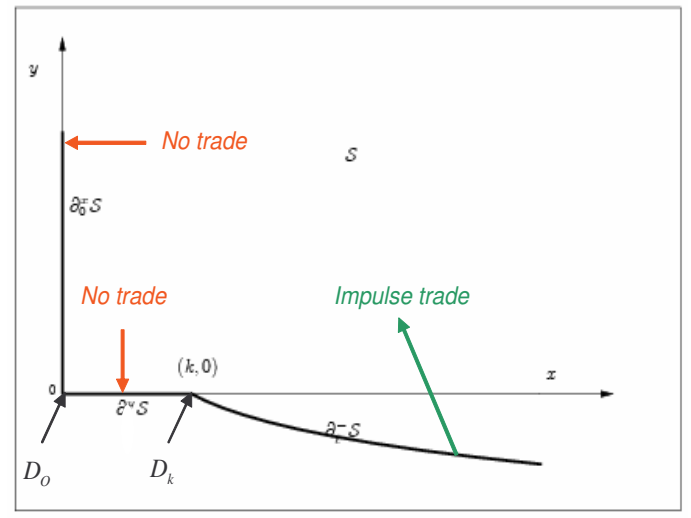

Figure 2: The solvency region when $p<k \lambda e$

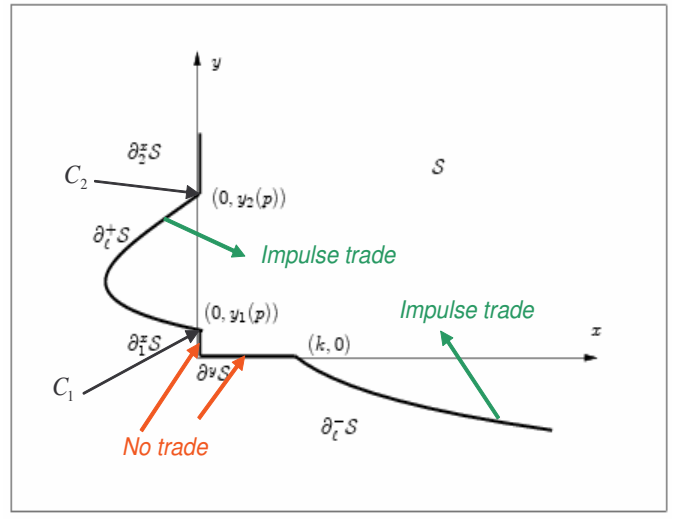

Figure 3: The solvency region when $p>k \lambda e$ 
Admissible controls. Given $t \in[0, T], z=(x, y, p) \in \overline{\mathcal{S}}$ and an initial state $Z_{t^{-}}=z$, we say that the impulse control strategy $\alpha=\left(\tau_{n}, \zeta_{n}\right)_{n \geq 1}$ is admissible if the process $Z_{s}$ $=\left(X_{s}, Y_{s}, P_{s}\right)$ given by (2.1)-(2.2)-(2.3) (with the convention $\left.\tau_{0}=t\right)$ lies in $\overline{\mathcal{S}}$ for all $s \in$ $[t, T]$. We denote by $\mathcal{A}(t, z)$ the set of all such policies. We shall see later that this set of admissible controls is nonempty for all $(t, z) \in[0, T] \times \overline{\mathcal{S}}$.

Remark 2.3 We recall that we do not allow intervention time at $T$, which is the liquidation date. This means that for all $\alpha \in \mathcal{A}(t, z)$, the associated state process $Z$ is continuous at T, i.e. $Z_{T^{-}}=Z_{T}$.

In the sequel, for $t \in[0, T], z=(x, y, p) \in \overline{\mathcal{S}}$, we also denote $X_{s}^{0, t, x}=x X_{s}^{0} / X_{t}^{0}, P_{s}^{0, t, p}=$ $p P_{s}^{0} / P_{t}^{0}, Z_{s}^{0, t, z}=\left(X_{s}^{0, t, x}, y, P_{s}^{0, t, p}\right), t \leq s \leq T$, the state process when no transaction (i.e. no impulse control) is applied between $t$ and $T$, i.e. the solution to :

$$
d Z_{s}^{0}=\left(\begin{array}{c}
r X_{s}^{0} \\
0 \\
b P_{s}^{0}
\end{array}\right) d s+\left(\begin{array}{c}
0 \\
0 \\
\sigma P_{s}^{0}
\end{array}\right) d W_{s},
$$

starting from $z$ at time $t$.

Investment problem. We consider an utility function $U$ from $\mathbb{R}_{+}$into $\mathbb{R}$, strictly increasing, concave and w.l.o.g. $U(0)=0$, and s.t. there exist $K \geq 0, \gamma \in[0,1)$ :

$$
U(w) \leq K w^{\gamma}, \quad \forall w \geq 0,
$$

We denote $U_{L}$ the function defined on $\overline{\mathcal{S}}$ by :

$$
U_{L}(z)=U(L(z))
$$

We study the problem of maximizing the expected utility from terminal liquidation wealth and we then consider the value function :

$$
v(t, z)=\sup _{\alpha \in \mathcal{A}(t, z)} \mathbb{E}\left[U_{L}\left(Z_{T}\right)\right], \quad(t, z) \in[0, T] \times \overline{\mathcal{S}} .
$$

Remark 2.4 We shall see later that for all $\alpha \in \mathcal{A}(t, z) \neq \emptyset, U_{L}\left(Z_{T}\right)$ is integrable so that the expectation in (2.7) is well-defined. Since $U$ is nonnegative and nondecreasing, we immediately get a lower bound for the value function :

$$
v(t, z) \geq U(0)=0, \quad \forall t \in[0, T], z=(x, y, p) \in \overline{\mathcal{S}} .
$$

We shall also see later that the value function $v$ is finite in $[0, T] \times \overline{\mathcal{S}}$ by providing a sharp upper bound.

Notice that in contrast to financial models without frictions or with proportional transaction costs, the dynamics of the state process $Z=(X, Y, P)$ is nonlinear and then the value function $v$ does not inherit the concavity property of the utility function. The solvency region is even not convex. In particular, one cannot derive as usual the continuity of the value function as a consequence of the concavity property. Moreover, for power-utility 
functions $U(w)=K w^{\gamma}$, the value function does not inherit the homogeneity property of the utility function.

We adopt a dynamic programming approach to study this utility maximization problem. Since the value function is not smooth and actually since we do not know its continuity a priori, we use the notion of constrained viscosity solutions in order to provide a weak formulation to the quasi-variational inequality arising from the dynamic programming principle and the boundary conditions coming from the solvency constraint. We end this section by recalling the dynamic programming principle for our stochastic control problem.

Dynamic PRogramming PRinciple. For all $(t, z) \in[0, T) \times \overline{\mathcal{S}}$, we have

$$
v(t, z)=\sup _{\alpha \in \mathcal{A}(t, z)} \mathbb{E}\left[v\left(\tau, Z_{\tau}\right)\right]
$$

where $\tau=\tau(\alpha)$ is any stopping time valued in $[t, T]$ depending on $\alpha$ in (2.8). The precise meaning is :

(i) for all $\alpha \in \mathcal{A}(t, z)$, for all $\tau \in \mathcal{T}_{t, T}$, set of stopping times valued in $[t, T]$ :

$$
\mathbb{E}\left[v\left(\tau, Z_{\tau}\right)\right] \leq v(t, z)
$$

(ii) for all $\varepsilon>0$, there exists $\hat{\alpha}^{\varepsilon} \in \mathcal{A}(t, z)$ s.t. for all $\tau \in \mathcal{T}_{t, T}$ :

$$
v(t, z) \leq \mathbb{E}\left[v\left(\tau, \hat{Z}_{\tau}^{\varepsilon}\right)\right]+\varepsilon .
$$

Here $\hat{Z}^{\varepsilon}$ denotes the state process starting from $z$ at $t$ and controlled by $\hat{\alpha}^{\varepsilon}$.

\section{Quasi-variational Hamilton-Jacobi-Bellman inequality and the main result}

In this section, we provide an heuristic derivation of the dynamic programming quasivariational inequality satisfied by the value function. We introduce some notations and state the main result. Starting from the initial state $Z_{t^{-}}=z=(x, y, p) \in \overline{\mathcal{S}}$ at time $t$, the investor has the following decisions :

(i) He immediately trades $\zeta$ shares of stock so that the state process jumps at time $t$ to $Z_{t}$ $=\left(X_{t}, Y_{t}, P_{t}\right)=(x-\theta(\zeta, p)-k, y+\zeta, Q(\zeta, p))$. We then introduce the impulse transaction function from $\overline{\mathcal{S}} \times \mathbb{R}$ into $\mathbb{R} \times \mathbb{R} \times \mathbb{R}_{+}^{*}$ :

$$
\Gamma(z, \zeta)=(x-\theta(\zeta, p)-k, y+\zeta, Q(\zeta, p))
$$

and the set of admissible transactions :

$$
\mathcal{C}(z)=\{\zeta \in \mathbb{R}: L(\Gamma(z, \zeta)) \geq 0\}
$$

By definition of the value function, we then have, as a consequence of the dynamic programming principle (2.9), applied to $\tau=t$ and by choosing any admissible control $\alpha \in$ $\mathcal{A}(t, z)$ with immediate impulse at $t$ with size $\zeta \in \mathcal{C}(z)$ :

$$
v(t, z) \geq v(t, \Gamma(z, \zeta)), \quad \forall \zeta \in \mathcal{C}(z) .
$$


We then define the impulse operator :

$$
\mathcal{H} \varphi(t, z)=\sup _{\zeta \in \mathcal{C}(z)} \varphi(t, \Gamma(z, \zeta))
$$

for any measurable function $\varphi$ on $[0, T] \times \overline{\mathcal{S}}$. If for some $z \in \overline{\mathcal{S}}$, the set $\mathcal{C}(z)$ is empty, we set by convention $\mathcal{H} \varphi(t, z)=-\infty$. From (3.1), we get :

$$
v \geq \mathcal{H} v, \quad \text { on }[0, T] \times \overline{\mathcal{S}}
$$

(ii) He does not trade during some small interval $[t, \tau]$ where the state price evolves according to :

$$
d Z_{s}=d\left(\begin{array}{c}
X_{s} \\
Y_{s} \\
P_{s}
\end{array}\right)=\left(\begin{array}{c}
r X_{s} \\
0 \\
b P_{s}
\end{array}\right) d s+\left(\begin{array}{c}
0 \\
0 \\
\sigma P_{s}
\end{array}\right) d W_{s} .
$$

By the dynamic programming principle (2.9), we have

$$
v(t, z) \geq \mathbb{E}\left[v\left(\tau, Z_{\tau}\right)\right],
$$

whenever the state process $Z_{s}=\left(X_{s}, Y_{s}, P_{s}\right)$ stays in $\overline{\mathcal{S}}$ during $[t, \tau]$, which is possible during some small period time $[t, \tau]$ if $z \in \mathcal{S}$. If $v$ were smooth, applying Itô's formula to $v\left(Z_{s}\right)$ between $t$ and $\tau$ and sending $\tau$ to $t$, we would obtain :

$$
-\frac{\partial v}{\partial t}-\mathcal{L} v \geq 0 \quad \text { on } \quad[0, T) \times \mathcal{S} .
$$

where $\mathcal{L}$ is the infinitesimal generator associated to the system (3.3) :

$$
\mathcal{L} v=r x \frac{\partial v}{\partial x}+b p \frac{\partial v}{\partial p}+\frac{1}{2} \sigma^{2} p^{2} \frac{\partial^{2} v}{\partial p^{2}} .
$$

Since one of the two decisions $(i)$ and $(i i)$ should be optimal, we obtain the quasivariational inequality satisfied by the value function :

$$
\min \left[-\frac{\partial v}{\partial t}-\mathcal{L} v, v-\mathcal{H} v\right]=0, \quad \text { on }[0, T) \times \mathcal{S} .
$$

The time-space liquidation solvency region $[0, T) \times \mathcal{S}$ is then divided into the following regions :

$\star$ A no-trade region

$$
\mathbf{N T}=\{(t, z) \in[0, T) \times \mathcal{S}: v(t, z)>\mathcal{H} v(t, z)\},
$$

$\star$ A trade region

$$
\mathbf{T}=\{(t, z) \in[0, T) \times \mathcal{S}: v(t, z)=\mathcal{H} v(t, z)\} .
$$

The rigorous characterization of the value function through the quasi-variational inequality (3.5) together with the boundary and terminal conditions will be proved in Section 5 by means of constrained viscosity solutions. Our main result is the following theorem, which follows from the results proved in Sections 4 and 5. 
Theorem 3.1 The value function $v$ is continuous on $[0, T) \times \mathcal{S}$ and is the unique $($ in $[0, T) \times$ $\mathcal{S})$ constrained viscosity solution to (3.5) satisfying the boundary and terminal condition:

$$
\begin{aligned}
& \lim _{\substack{\left(t^{\prime}, z^{\prime}\right) \rightarrow(t, z) \\
z^{\prime} \in \mathcal{S}}} v\left(t^{\prime}, z^{\prime}\right)=0, \quad \forall(t, z) \in[0, T) \times D_{0} \\
& \lim _{\substack{\left.t, z^{\prime}\right) \rightarrow(T, z) \\
t<T, z^{\prime} \in \mathcal{S}}} v\left(t, z^{\prime}\right)=\max \left[U_{L}(z), \mathcal{H} U_{L}(z)\right], \quad \forall z \in \overline{\mathcal{S}},
\end{aligned}
$$

and the growth condition:

$$
|v(t, z)| \leq K\left(1+\left(x+\frac{p}{\lambda}\right)\right)^{\gamma}, \quad \forall(t, z) \in[0, T) \times \mathcal{S}
$$

for some positive constant $K<\infty$.

\section{Properties of the value function}

\subsection{Some properties on the impulse transactions set}

As mentioned in Section 1, it is not clear why the set of admissible controls is not empty.

To prove this fact, we need to derive some preliminary remarks on the set of admissible transactions $\mathcal{C}(z)$. Suppose the current position of the investor is a point $z=(x, y, p) \in \overline{\mathcal{S}}$. If he makes a transaction of size $\zeta$ at that time, then after the transaction the new position is given by $\Gamma(z, \zeta)=\left(x^{\prime}, y^{\prime}, p^{\prime}\right)$ with

$$
\begin{aligned}
x^{\prime} & =x-\theta(\zeta, p)-k=x-p \zeta e^{\lambda \zeta}-k \\
y^{\prime} & =y+\zeta \\
p^{\prime} & =Q(\zeta, p)=p e^{\lambda \zeta} .
\end{aligned}
$$

Hence, the net wealth value with liquidation is given by :

$$
\begin{aligned}
L_{0}(\Gamma(z, \zeta))=x^{\prime}+\ell\left(y^{\prime}, p^{\prime}\right)-k & =x-p \zeta e^{\lambda \zeta}-k+p e^{\lambda \zeta}(y+\zeta) e^{-\lambda(y+\zeta)}-k \\
& =x+\ell(y, p)-k+p \zeta\left(e^{-\lambda y}-e^{\lambda \zeta}\right)-k \\
& =L_{0}(z)+p g(y, \zeta)-k,
\end{aligned}
$$

with

$$
g(y, \zeta)=\zeta\left(e^{-\lambda y}-e^{\lambda \zeta}\right)
$$

Globally, the net wealth after transaction is equal to :

$$
L(\Gamma(z, \zeta))=\max \left[L_{0}(\Gamma(z, \zeta)), L_{1}(\Gamma(z, \zeta))\right] 1_{y+\zeta \geq 0}+L_{0}(\Gamma(z, \zeta)) 1_{y+\zeta<0}
$$

We then see that, in contrast with transaction costs models, the net wealth may grow after some transaction. We first prove the following elementary lemma. 
Lemma 4.1 For any $y \in \mathbb{R}$, there exists an unique $\bar{\zeta}(y) \in \mathbb{R}$ s.t.

$$
\bar{g}(y):=\max _{\zeta \in \mathbb{R}} g(y, \zeta)=\bar{\zeta}(y)\left(e^{-\lambda y}-e^{\lambda \bar{\zeta}(y)}\right) .
$$

(i) The function $\bar{\zeta}$ is differentiable, decreasing one to one from $\mathbb{R}$ into $(-1 / \lambda, \infty)$ with $\bar{\zeta}(0)$ $=0$, and

$$
0<\bar{\zeta}(y)<-y \text { if } y<0 \text { and }-y<\bar{\zeta}(y)<0 \text { if } y>0 .
$$

(ii) The function $\bar{g}$ is differentiable, decreasing on $(-\infty, 0)$, increasing on $(0, \infty)$, with $\bar{g}(0)$ $=0, \lim _{y \rightarrow-\infty} \bar{g}(y)=\infty, \lim _{y \rightarrow \infty} \bar{g}(y)=e^{-1} / \lambda$, and for all $p>0$,

$$
\ell(y, p)+p \bar{g}(y)<0 \quad \text { if } y<0 \quad \text { and } \quad-\ell(y, p)+p \bar{g}(y)<0 \quad \text { if } 0<y \leq \frac{1}{\lambda} .
$$

Proof. (i) For fixed $y$, a straightforward study of the differentiable function $\zeta \rightarrow g_{y}(\zeta):=$ $g(y, \zeta)$ shows that there exists an unique $\bar{\zeta}(y) \in \mathbb{R}$ such that :

$$
\begin{aligned}
G(y, \bar{\zeta}(y))=g_{y}^{\prime}(\bar{\zeta}(y)) & =e^{-\lambda y}-e^{\lambda \bar{\zeta}(y)}(1+\lambda \bar{\zeta}(y))=0, \\
g_{y}^{\prime}(\zeta)>(\text { resp. }<) 0 & \Longleftrightarrow \zeta<(\text { resp. }>) \bar{\zeta}(y)
\end{aligned}
$$

This proves that $g_{y}$ is increasing on $(-\infty, \bar{\zeta}(y))$ and decreasing on $(\bar{\zeta}(y), \infty)$ with

$$
\max _{\zeta \in \mathbb{R}} g_{y}(\zeta)=g_{y}(\bar{\zeta}(y)):=\bar{g}(y)
$$

i.e. (4.3). Since $g_{y}^{\prime}(-1 / \lambda)=e^{-\lambda y}>0$, we notice that $\bar{\zeta}(y)$ is valued in $(-1 / \lambda, \infty)$ for all $y$ $\in \mathbb{R}$. Moreover, since the differentiable function $(y, \zeta) \rightarrow G(y, \zeta):=g_{y}^{\prime}(\zeta)$ is decreasing in $y$ on $\mathbb{R}: \frac{\partial G}{\partial y}<0$ and decreasing in $\zeta$ on $(-1 / \lambda, \infty): \frac{\partial G}{\partial \zeta}<0$, we derive by the implicit theorem that $\bar{\zeta}(y)$ is a differentiable decreasing function on $\mathbb{R}$. Since $G(y,-1 / \lambda)=e^{-\lambda y}$ goes to zero as $y$ goes to infinity, we also obtain that $\bar{\zeta}(y)$ goes to $-1 / \lambda$ as $y$ goes to infinity. By noting that for all $\zeta, G(y, \zeta)$ goes to $\infty$ when $y$ goes to $-\infty$, we deduce that $\bar{\zeta}(y)$ goes to $\infty$ as $y$ goes to $-\infty$. Since $G(0,0)=0$, we also have $\bar{\zeta}(0)=0$. Notice also that $G(y,-y)$ $=\lambda y e^{-\lambda y}$ : hence, when $y<0, G(y,-y)<0=G(y, \bar{\zeta}(y))$ so that $\bar{\zeta}(y)<-y$, and when $y$ $>0, G(y,-y)>0=G(y, \bar{\zeta}(y))$ so that $\bar{\zeta}(y)>-y$.

(ii) By the envelope theorem, the function $\bar{g}$ defined by $\bar{g}(y)=\max _{\zeta \in \mathbb{R}} g(y, \zeta)=$ $g(y, \bar{\zeta}(y))$ is differentiable on $\mathbb{R}$ with

$$
\bar{g}^{\prime}(y)=\frac{\partial g}{\partial y}(y, \bar{\zeta}(y))=-\lambda \bar{\zeta}(y) e^{-\lambda \bar{\zeta}(y)}, \quad y \in \mathbb{R} .
$$

Since $\bar{\zeta}(y)>($ resp. $<) 0$ iff $y<($ resp. $>$ ) 0 with $\bar{\zeta}(0)=0$, we deduce the decreasing (resp. increasing) property of $\bar{g}$ on $(-\infty, 0)$ (resp. $(0, \infty))$ with $\bar{g}(0)=0$. Since $\bar{\zeta}(y)$ converges to $-1 / \lambda$ as $y$ goes to infinity, we immediately see from expression (4.3) of $\bar{g}$ that $\bar{g}(y)$ converges to $e^{-1} / \lambda$ as $y$ goes to infinity. For $y<0$ and by taking $\zeta=-y / 2$ in the maximum in (4.3), we have $\bar{g}(y) \geq-y\left(e^{-\lambda y}-e^{-\lambda y / 2}\right) / 2$, which shows that $\bar{g}(y)$ goes to infinity as $y$ goes to $-\infty$. When $y<0$, we have $0<\bar{\zeta}(y)<-y$, and thus by (4.3), we get :

$$
\bar{g}(y)<-y\left(e^{-\lambda y}-e^{\lambda \bar{\zeta}(y)}\right)
$$


and so $\ell(y, p)+p \bar{g}(y)<p y e^{\lambda \bar{\zeta}(y)}<0$ for all $p>0$. When $y>0$, we have $\bar{\zeta}(y)<0$ and thus by (4.3), we get : $\bar{g}(y)<-\bar{\zeta}(y) e^{\lambda \bar{\zeta}(y)}$. Now, since the function $\zeta \mapsto-\zeta e^{\lambda \zeta}$ is decreasing on $[-1 / \lambda, 0]$, we have for all $0<y \leq 1 / \lambda,-1 / \lambda \leq-y<\bar{\zeta}(y)$ and so

$$
\bar{g}(y)<y e^{-\lambda y} .
$$

This proves $p \bar{g}(y) \leq \ell(y, p)$ for all $0<y \leq 1 / \lambda$ and $p>0$.

We next state the following useful result on the set of admissible transactions.

Lemma 4.2 For all $z \in \overline{\mathcal{S}}$, the set $\mathcal{C}(z)$ is compact, eventually empty. We have:

$$
\begin{aligned}
\mathcal{C}(z) & =\emptyset \quad \text { if } z \in \partial^{y} \mathcal{S} \cup \partial_{0}^{x} \mathcal{S} \cup \partial_{1}^{x} \mathcal{S}, \\
-\frac{1}{\lambda} \in \mathcal{C}(z) & \subset(-y, 0) \quad \text { if } z \in \partial_{2}^{x} \mathcal{S}, \\
-y \in \mathcal{C}(z) & \subset \begin{cases}{[0,-y]} & \text { if } z \in \partial_{\ell}^{-} \mathcal{S} \\
{[-y, 0)} & \text { if } z \in \partial_{\ell}^{+} \mathcal{S}\end{cases}
\end{aligned}
$$

Moreover,

$$
\begin{aligned}
& \mathcal{C}(z)=\{-y\} \quad \text { if } z \in\left(\partial_{\ell}^{-} \mathcal{S} \cup \partial_{\ell}^{+, \lambda} \mathcal{S}\right) \cap \mathcal{N}_{\ell} \\
& \bar{\zeta}(y) \in \mathcal{C}(z) \quad \text { if } z \in\left(\partial_{\ell}^{-} \mathcal{S} \cup \partial_{\ell}^{+} \mathcal{S}\right) \cap\left(\overline{\mathcal{S}} \backslash \mathcal{N}_{\ell}\right)
\end{aligned}
$$

where

$$
\begin{aligned}
\partial_{\ell}^{+, \lambda} \mathcal{S} & =\partial_{\ell}^{+} \mathcal{S} \cap\left\{z \in \overline{\mathcal{S}}: y \leq \frac{1}{\lambda}\right\} \\
\mathcal{N}_{\ell} & =\{z \in \overline{\mathcal{S}}: p \bar{g}(y)<k\} .
\end{aligned}
$$

Proof. For any $z \in \overline{\mathcal{S}}$, we write $\mathcal{C}(z)=\mathcal{C}_{0}(z) \cup \mathcal{C}_{1}(z)$ where $\mathcal{C}_{0}(z)=\left\{\zeta \in \mathbb{R}: L_{0}(\Gamma(z, \zeta)) \geq\right.$ $0\}$ and $\mathcal{C}_{1}(z)=\left\{\zeta \in \mathbb{R}: L_{1}(\Gamma(z, \zeta)) \geq 0, y+\zeta \geq 0\right\}$. From (4.1) and by noting that the function $\zeta \mapsto p g(y, \zeta)$ goes to $-\infty$ as $|\zeta|$ goes to infinity, we see that $\mathcal{C}_{0}(z)$ is bounded. Since the function $\zeta \mapsto p \theta(\zeta, p)$ goes to infinity as $\zeta$ goes to infinity, we also see that $\mathcal{C}_{1}(z)$ is bounded. Hence, $\mathcal{C}(z)$ is bounded. Moreover, for any $z=(x, y, p) \in \overline{\mathcal{S}}$, the function $\zeta \mapsto L(\Gamma(z, \zeta))$ is uppersemicontinuous : it is indeed continuous on $\mathbb{R} \backslash\{-y\}$ and uppersemicontinuous on $-y$. This implies the closure property and then the compactness of $\mathcal{C}(z)$.

$\star$ Fix some arbitrary $z \in \partial^{y} \mathcal{S}$. Then, for any $\zeta \in \mathbb{R}$, we have $L_{0}(\Gamma(z, \zeta))=x-k+p g(0, \zeta)-k$. Since $g(0, \zeta) \leq 0$ for all $\zeta \in \mathbb{R}$ and $x \leq k$, we see that $L(\Gamma(z, \zeta))<0$ for all $\zeta \in \mathbb{R}$. On the other hand, we have $L_{1}(\Gamma(z, \zeta))=x-\theta(\zeta, p)-k$. Since $\theta(\zeta, p) \geq 0$ for all $\zeta \geq 0$, and recalling that $x<k$, we also see that $L_{1}(\Gamma(z, \zeta))=x-\theta(\zeta, p)-k<0$ for all $\zeta \geq 0$. Therefore $L(\Gamma(z, \zeta))<0$ for all $\zeta \in \mathbb{R}$ and so $\mathcal{C}(z)$ is empty.

$\star$ Fix some arbitrary $z \in \partial_{0}^{x} \mathcal{S}$. Then, for any $\zeta \in \mathbb{R}$, we have $L_{0}(\Gamma(z, \zeta))=\ell(y, p)-k+$ $p g(y, \zeta)-k$. Now, we recall from Remark 2.2 that $\ell(y, p) \leq p /(\lambda e)<k$. Moreover, by Lemma 4.1, we have $p g(y, \zeta) \leq p \bar{g}(y) \leq p /(\lambda e)<k$. This implies $L_{0}(\Gamma(z, \zeta))<0$ for any $\zeta$ $\in \mathbb{R}$. On the other hand, we have $L_{1}(\Gamma(z, \zeta))=-\theta(\zeta, p)-k$. Since $\theta(\zeta, p) \geq-p /(\lambda e)$ for all $\zeta \in \mathbb{R}$, we get $L_{1}(\Gamma(z, \zeta)) \leq p /(\lambda e)-k<0$. Therefore $\mathcal{C}(z)$ is empty. 
$\star$ Fix some arbitrary $z \in \partial_{1}^{x} \mathcal{S}$. Then, for any $\zeta \in \mathbb{R}$, we have $L_{0}(\Gamma(z, \zeta))=\ell(y, p)-k+$ $p g(y, \zeta)-k$. Now, we recall from Remark 2.2 that $\ell(y, p)<k$. Moreover, since $0<y \leq$ $1 / \lambda$, we get from Lemma 4.1: $p g(y, \zeta) \leq p \bar{g}(y)<\ell(y, p)<k$ for all $\zeta \in \mathbb{R}$. This implies $L_{0}(\Gamma(z, \zeta))<0$ for any $\zeta \in \mathbb{R}$. On the other hand, we have $L_{1}(\Gamma(z, \zeta))=-\theta(\zeta, p)-k$. Since the function $\zeta \mapsto \theta(\zeta, p)$ is increasing on $[-1 / \lambda, \infty)$ and $y<1 / \lambda$, we have for all $\zeta$ $\geq-y, \theta(\zeta, p) \geq \theta(-y, p)=-\ell(y, p)$, and so $-\theta(\zeta, p)-k \leq \ell(y, p)-k<0$. This implies $L_{1}(\Gamma(z, \zeta))<0$ for all $\zeta \in \mathbb{R}$ and thus $\mathcal{C}(z)$ is empty.

$\star$ Fix some arbitrary $z \in \partial_{2}^{x} \mathcal{S}$. Then for $\zeta=-1 / \lambda$, we have $\theta(\zeta, p)=-p /(\lambda e)$ and $y+\zeta$ $>0$ (see Remark 2.2). Hence, $L(\Gamma(z,-1 / \lambda)) \geq L_{1}(\Gamma(z,-1 / \lambda)) \geq 0$ and so $-1 / \lambda \in \mathcal{C}(z)$. Moreover, take some arbitrary $\zeta \in \mathcal{C}(z)=\mathcal{C}_{0}(z) \cup \mathcal{C}_{1}(z)$. In the case where $\zeta \in \mathcal{C}_{0}(z)$, i.e. $L_{0}(\Gamma(z, \zeta))=\ell(y, p)-k+p g(y, \zeta)-k \geq 0$, and recalling that $\ell(y, p)<k$, we must have necessarily $g(y, \zeta)>0$. This implies $-y<\zeta<0$. Similarly, when $\zeta \in \mathcal{C}_{1}(z)$, i.e. $-\theta(\zeta, p)-k \geq 0$ and $y+\zeta \geq 0$, we must have $-y<\zeta<0$. Therefore, $\mathcal{C}(z) \subset(-y, 0)$.

$\star$ Fix some arbitrary $z \in \partial_{\ell}^{-} \mathcal{S} \cup \partial_{\ell}^{+} \mathcal{S}$. Then we have $L(\Gamma(z,-y))=L_{1}(\Gamma(z,-y))=0$, which shows that $\zeta=-y \in \mathcal{C}(z)$. Consider now the case where $z \in \partial_{\ell}^{-} \mathcal{S} \cup \partial_{\ell}^{+, \lambda} \mathcal{S}$. We claim that $\mathcal{C}_{1}(z)=\{-y\}$. Indeed, take some $\zeta \in \mathcal{C}_{1}(z)$, i.e. $x-\theta(\zeta, p)-k \geq 0$ and $y+\zeta$ $\geq 0$. Then, $\theta(\zeta, p) \geq \theta(-y, p)=-\ell(y, p)$ (since $\zeta \mapsto \theta(\zeta, p)$ is increasing on $[-1 / \lambda, \infty)$ and $-y \geq-1 / \lambda)$ and so $0 \leq x-\theta(\zeta, p)-k \leq x+\ell(y, p)-k=0$. Hence, we must have $\zeta=-y$. Take now some arbitrary $\zeta \in \mathcal{C}_{0}(z)$. Hence, $L_{0}(\Gamma(z, \zeta))=p g(y, \zeta)-k \geq 0$, and we must have necessarily $g(y, \zeta) \geq 0$. Since $y \leq 0$, this implies $0 \leq \zeta \leq-y$. We have then showed that $\mathcal{C}(z) \subset[-y, 0]$. Consider now the case where $z \in \partial_{\ell}^{+} \mathcal{S}$ and take some arbitrary $\zeta \in$ $\mathcal{C}(z)=\mathcal{C}_{0}(z) \cup \mathcal{C}_{1}(z)$. If $\zeta \in \mathcal{C}_{0}(z)$, then similarly as above, we must have $p g(y, \zeta)-k \geq 0$. Since $y>0$, this implies $-y \leq \zeta<0$. If $\zeta \in \mathcal{C}_{1}(z)$, i.e. $x-\theta(\zeta, p)-k \geq 0$ and $y+\zeta \geq 0$, and recalling that $x<k$, we must have also $-y \leq \zeta<0$. We have then showed that $\mathcal{C}(z)$ $\subset[-y, 0)$.

Notice that for $z \in\left(\partial_{\ell}^{-} \mathcal{S} \cup \partial_{\ell}^{+} \mathcal{S}\right) \cap \mathcal{N}_{\ell}$, we have $L_{0}(\Gamma(z, \zeta)) \leq p \bar{g}(y)-k<0$ for all $\zeta \in$ $\mathbb{R}$. Hence, $\mathcal{C}_{0}(z)=\emptyset$. We have already seen that $\mathcal{C}_{1}(z)=\{-y\}$ when $z \in \partial_{\ell}^{-} \mathcal{S} \cup \partial_{\ell}^{+, \lambda} \mathcal{S}$ and so $\mathcal{C}(z)=\{-y\}$ when $z \in\left(\partial_{\ell}^{-} \mathcal{S} \cup \partial_{\ell}^{+, \lambda} \mathcal{S}\right) \cap \mathcal{N}_{\ell}$. Finally, when $z \in\left(\partial_{\ell}^{-} \mathcal{S} \cup \partial_{\ell}^{+} \mathcal{S}\right) \cap\left(\overline{\mathcal{S}} \backslash \mathcal{N}_{\ell}\right)$, we have $L_{0}(\Gamma(z, \bar{\zeta}(u)))=p \bar{g}(y)-k \geq 0$, which shows that $\bar{\zeta}(y) \in \mathcal{C}(z)$.

Remark 4.1 Actually, we have a more precise result on the compactness result of $\mathcal{C}(z)$. Let $z \in \overline{\mathcal{S}}$ and $\left(z_{n}\right)_{n}$ be a sequence in $\overline{\mathcal{S}}$ converging to $z$. Consider any sequence $\left(\zeta_{n}\right)_{n}$ with $\zeta_{n} \in \mathcal{C}\left(z_{n}\right)$, i.e. $L\left(\Gamma\left(z_{n}, \zeta_{n}\right)\right) \geq 0$ :

$$
\begin{aligned}
& \max \left[L_{0}\left(z_{n}\right)+p_{n} g\left(y_{n}, \zeta_{n}\right)-k, x-\theta\left(\zeta_{n}, p_{n}\right)-k\right] 1_{y_{n}+\zeta_{n} \geq 0} \\
&+\left[L_{0}\left(z_{n}\right)+p_{n} g\left(y_{n}, \zeta_{n}\right)-k\right] 1_{y_{n}+\zeta_{n}<0} \geq 0 .
\end{aligned}
$$

Since $g(y, \zeta)$ and $-\theta(\zeta, p)$ goes to $-\infty$ as $\zeta$ goes to infinity, and $g(y, \zeta)$ goes to $-\infty$ as $\zeta$ goes to $-\infty$, this proves that the sequence $\left(\zeta_{n}\right)$ is bounded. Hence, up to a subsequence, $\left(\zeta_{n}\right)$ converges to some $\zeta \in \mathbb{R}$. Since the function $L$ is uppersemicontinuous, we see that the limit $\zeta$ satisfies : $L(\Gamma(z, \zeta)) \geq 0$, i.e. $\zeta$ lies in $\mathcal{C}(z)$.

Corollary 4.1 For all $(t, z) \in[0, T) \times \overline{\mathcal{S}}$, we have $\mathcal{A}(t, z) \neq \emptyset$. 
Proof. By continuity of the process $Z_{s}^{0, t, z}, t \leq s \leq T$, it is clear that it suffices to prove $\mathcal{A}(t, z) \neq \emptyset$ for any $t \in[0, T) \times \partial \mathcal{S}$. Fix now some arbitrary $t \in[0, T)$. From Lemma 4.2, the set of admissible transactions $\mathcal{C}(z)$ contains at least one nonzero element for any $z \in$ $\partial_{2}^{x} \mathcal{S} \cup \partial_{\ell}^{+} \mathcal{S} \cup \partial_{\ell}^{-} \mathcal{S} \backslash D_{k}$. So once the state process reaches this boundary part, it is possible to jump inside the open solvency region $\mathcal{S}$. Hence, we only have to check that $\mathcal{A}(t, z)$ is nonempty when $z \in \partial_{0}^{x} \mathcal{S} \cup \partial_{1}^{x} \mathcal{S} \cup \partial^{y} \mathcal{S} \cup D_{k}$. This is clear when $z \in \partial^{y} \mathcal{S} \cup D_{k}$ : indeed, by doing nothing the state process $Z_{s}=Z_{s}^{0, t, z}=\left(x e^{r(s-t)}, 0, P_{s}^{0, t, p}\right), t \leq s \leq T$, obviously stays in $\overline{\mathcal{S}}$, since $x \geq 0$ and so $L_{1}\left(Z_{s}\right) \geq 0$ for all $t \leq s \leq T$. Similarly, when $z \in \partial_{0}^{x} \mathcal{S} \cup$ $\partial_{1}^{x} \mathcal{S}$, by doing nothing the state process $Z_{s}=Z_{s}^{0, t, z}=\left(0, y, P_{s}^{0, t, p}\right), t \leq s \leq T$, also stays in $\overline{\mathcal{S}}$ since $y \geq 0$ and so $L_{1}\left(Z_{s}\right) \geq 0$ for all $t \leq s \leq T$.

We next turn to the finiteness of the value function, which is not trivial due to the impulse control. As mentioned above, since the net wealth may grow after transaction due to the nonlinearity of the liquidation function, one cannot bound the value function $v$ by the value function of the Merton problem with liquidated net wealth. We then introduce a suitable "linearization" of the net wealth by defining the following functions on $\overline{\mathcal{S}}$ :

$$
\begin{aligned}
& \tilde{L}(z)=x+\frac{p}{\lambda}\left(1-e^{-\lambda y}\right), \quad z=(x, y, p) \in \overline{\mathcal{S}} \\
& \bar{L}(z)=x+\frac{p}{\lambda}, \quad z=(x, y, p) \in \overline{\mathcal{S}} .
\end{aligned}
$$

Lemma 4.3 For all $z=(x, y, p) \in \overline{\mathcal{S}}$, we have :

$$
0 \leq L(z) \leq \tilde{L}(z) \leq \bar{L}(z)
$$

and for all $\zeta \in \mathcal{C}(z)$

$$
\begin{aligned}
& \tilde{L}(\Gamma(z, \zeta)) \leq \tilde{L}(z)-k \\
& \bar{L}(\Gamma(z, \zeta)) \leq \bar{L}(z)-k .
\end{aligned}
$$

In particular, we have $\mathcal{C}(z)=\emptyset$ for all $z \in \tilde{\mathcal{N}}:=\{z \in \mathcal{S}: \tilde{L}(z)<k\}$.

Proof. 1) The inequality $\tilde{L} \leq \bar{L}$ is clear. Notice that for all $y \in \mathbb{R}$, we have

$$
0 \leq 1-e^{-\lambda y}-\lambda y e^{-\lambda y}
$$

This immediately implies for all $z=(x, y, p) \in \overline{\mathcal{S}}$,

$$
L_{0}(z) \leq \tilde{L}(z)
$$

If $y \geq 0$, we obviously have $L_{1}(z)=x \leq \tilde{L}(z)$ and so $L(z) \leq \tilde{L}(z)$. If $y<0$, then $L(z)=$ $L_{0}(z) \leq \tilde{L}(z)$ by $(4.8)$.

2) For any $z=(x, y, p) \in \overline{\mathcal{S}}$ and $\zeta \in \mathbb{R}$, a straightforward computation shows that

$$
\begin{aligned}
\tilde{L}(\Gamma(z, \zeta)) & =\tilde{L}(z)-k+\frac{p}{\lambda}\left(e^{\lambda \zeta}-1-\lambda \zeta e^{\lambda \zeta}\right) \\
& \leq \tilde{L}(z)-k
\end{aligned}
$$


from (4.7). Similarly, we have

$$
\bar{L}(\Gamma(z, \zeta))=\bar{L}(z)-k+\frac{p}{\lambda}\left(e^{\lambda \zeta}-1-\lambda \zeta e^{\lambda \zeta}\right) \leq \bar{L}(z)-k
$$

Finally, if $z \in \tilde{N} c$, we have from $(4.6), \tilde{L}(\Gamma(z, \zeta))<0$ for all $\zeta \in \mathcal{C}(z)$, which shows with (4.4) that $\mathcal{C}(z)=\emptyset$.

As a first direct corollary, we check that the no-trade region is not empty.

Corollary 4.2 We have $\mathbf{N T} \neq \emptyset$. More precisely, for each $t \in[0, T)$, the $t$-section of $\mathbf{N T}$, i.e. $\mathbf{N T}(t)=\{z \in \mathcal{S}:(t, z) \in \mathbf{N T}\}$ contains the nonempty subset $\tilde{\mathcal{N}}$ of $\mathcal{S}$.

Proof. This follows from the fact that for any $z$ lying in the nonempty set $\tilde{\mathcal{N}}$ of $\mathcal{S}$, we have $\mathcal{C}(z)=\emptyset$. In particular, $\mathcal{H} v(t, z)=-\infty<v(t, z)$ for $(t, z) \in[0, T) \times \tilde{\mathcal{N}}$.

As a second corollary, we have the following uniform bound on the controlled state process.

Corollary 4.3 For any $(t, z) \in[0, T] \times \overline{\mathcal{S}}$, we have almost surely for all $t \leq s \leq T$ :

$$
\begin{aligned}
\sup _{\alpha \in \mathcal{A}(t, z)} L\left(Z_{s}\right) \leq \sup _{\alpha \in \mathcal{A}(t, z)} \tilde{L}\left(Z_{s}\right) & \leq \tilde{L}\left(Z_{s}^{0, t, z}\right)=X_{s}^{0, t, x}+\frac{P_{s}^{0, t, p}}{\lambda}\left(1-e^{-\lambda y}\right), \\
\sup _{\alpha \in \mathcal{A}(t, z)} L\left(Z_{s}\right) \leq \sup _{\alpha \in \mathcal{A}(t, z)} \bar{L}\left(Z_{s}\right) & \leq \bar{L}\left(Z_{s}^{0, t, z}\right)=X_{s}^{0, t, x}+\frac{P_{s}^{0, t, p}}{\lambda} \\
\sup _{\alpha \in \mathcal{A}(t, z)}\left|X_{s}\right| & \leq \frac{e}{e-1} \bar{L}\left(Z_{s}^{0, t, z}\right) \\
\sup _{\alpha \in \mathcal{A}(t, z)} P_{s} & \leq \frac{\lambda e}{e-1} \bar{L}\left(Z_{s}^{0, t, z}\right) .
\end{aligned}
$$

Proof. Fix $(t, z) \in[0, T] \times \overline{\mathcal{S}}$ and consider for any $\alpha \in \mathcal{A}(t, z)$, the process $\tilde{L}\left(Z_{s}\right), t \leq s \leq T$, which is nonnegative by (4.4). When a transaction occurs at time $s$, we deduce from (4.5) that the variation $\Delta \tilde{L}\left(Z_{s}\right)=\tilde{L}\left(Z_{s}\right)-\tilde{L}\left(Z_{s^{-}}\right)$is always negative : $\Delta \tilde{L}\left(Z_{s}\right) \leq-k \leq 0$. Therefore, the process $\tilde{L}\left(Z_{s}\right)$ is smaller than its continuous part:

$$
L\left(Z_{s}\right) \leq \tilde{L}\left(Z_{s}\right) \leq \tilde{L}\left(Z_{s}^{0, t, z}\right), \quad t \leq s \leq T, \text { a.s. }
$$

which proves (4.9) from the arbitrariness of $\alpha$. Relation (4.10) is proved similarly.

From the second inequality in (4.10), we have for all $\alpha \in \mathcal{A}(t, z)$ :

$$
\begin{aligned}
X_{s} & \leq \bar{L}\left(Z_{s}^{0, t, z}\right)-\frac{P_{s}}{\lambda}, \quad t \leq s \leq T, \text { a.s. } \\
& \leq \bar{L}\left(Z_{s}^{0, t, z}\right), \quad t \leq s \leq T, \text { a.s. }
\end{aligned}
$$

By definition of $L$ and using (4.14), we have :

$$
\begin{aligned}
0 \leq L\left(Z_{s}\right) & \leq \max \left(\bar{L}\left(Z_{s}^{0, t, z}\right)-\frac{P_{s}}{\lambda}\left(1-\lambda Y_{s} e^{-\lambda Y_{s}}\right), \bar{L}\left(Z_{s}^{0, t, z}\right)-\frac{P_{s}}{\lambda}\right) \\
& \leq \bar{L}\left(Z_{s}^{0, t, z}\right)-\frac{P_{s}}{\lambda}\left(1-\frac{1}{e}\right), \quad t \leq s \leq T, \text { a.s. }
\end{aligned}
$$


since the function $y \mapsto \lambda y e^{-\lambda y}$ is upper bounded by $1 / e$. We then deduce

$$
P_{s} \leq \frac{\lambda e}{e-1} \bar{L}\left(Z_{s}^{0, t, z}\right), \quad t \leq s \leq T \text {, a.s. }
$$

and so (4.12) from the arbitrariness of $\alpha$. By recalling that $X_{s}+P_{s} / \lambda \geq 0$ and using (4.16), we get

$$
-\frac{e}{e-1} \bar{L}\left(Z_{s}^{0, t, z}\right) \leq X_{s}, \quad t \leq s \leq T, \text { a.s. }
$$

By combining with (4.15) and from the arbitrariness of $\alpha$, we obtain (4.11).

As a third direct corollary, we state that the number of intervention times is finite. More precisely, we have the following result :

Corollary 4.4 For any $(t, z) \in[0, T] \times \overline{\mathcal{S}}, \alpha=\left(\tau_{n}, \zeta_{n}\right) \in \mathcal{A}(t, z)$, the number of intervertion times strictly between $t$ and $T$ is finite a.s. :

$$
\begin{aligned}
N_{t}(\alpha) & :=\operatorname{Card}\left\{n: t<\tau_{n}<T\right\} \\
& \leq \frac{1}{k}\left[\bar{L}\left(Z_{t}\right)-\bar{L}\left(Z_{T^{-}}\right)+\int_{t}^{T}\left(r X_{s}+\frac{P_{s}}{\lambda}\right) d s+\int_{t}^{T} \frac{\sigma}{\lambda} P_{s} d W_{s}\right]<\infty \text { a.s.(4. }
\end{aligned}
$$

Proof. Fix some $(t, z) \in[0, T] \times \overline{\mathcal{S}}$ and $\alpha \in \mathcal{A}(t, z)$, and consider $Z_{s}=\left(X_{s}, Y_{s}, P_{s}\right)$, $t \leq s \leq T$, the associated controlled state process. By applying Itô's formula to $\bar{L}\left(Z_{s}\right)=$ $X_{s}+P_{s} / \lambda$ between $t$ and $T$, we have :

$$
\begin{aligned}
0 \leq \bar{L}\left(Z_{T^{-}}\right) & =\bar{L}\left(Z_{t}\right)+\int_{t}^{T}\left(r X_{s}+\frac{P_{s}}{\lambda}\right) d s+\int_{t}^{T} \frac{\sigma}{\lambda} P_{s} d W_{s}+\sum_{t<s<T} \Delta L\left(Z_{s}\right) \\
& \leq \bar{L}\left(Z_{t}\right)+\int_{t}^{T}\left(r X_{s}+\frac{P_{s}}{\lambda}\right) d s+\int_{t}^{T} \frac{\sigma}{\lambda} P_{s} d W_{s}-k N_{t}(\alpha),
\end{aligned}
$$

by (4.6). We deduce the required result :

$$
N_{t}(\alpha) \leq \frac{1}{k}\left[\bar{L}\left(Z_{t}\right)-\bar{L}\left(Z_{T^{-}}\right)+\int_{t}^{T}\left(r X_{s}+\frac{P_{s}}{\lambda}\right) d s+\int_{t}^{T} \frac{\sigma}{\lambda} P_{s} d W_{s}\right]<\infty \text { a.s. }
$$

\subsection{Bound on the value function}

We now give a sharp upper bound on the value function.

Proposition 4.1 For all $t \in[0, T], z=(x, y, p) \in \overline{\mathcal{S}}$, we have

$$
\sup _{\alpha \in \mathcal{A}(t, z)} U_{L}\left(Z_{T}\right) \leq U\left(\tilde{L}\left(Z_{T}^{0, t, z}\right)\right) \in L^{1}(\mathbb{P}) .
$$

In particular, the family $\left\{U_{L}\left(Z_{T}\right), \alpha \in \mathcal{A}(t, z)\right\}$ is uniformly integrable and we have

$$
v(t, z) \leq v_{0}(t, z):=\mathbb{E}\left[U\left(\tilde{L}\left(Z_{T}^{0, t, z}\right)\right)\right], \quad(t, z) \in[0, T] \times \overline{\mathcal{S}},
$$


with

$$
v_{0}(t, z) \leq K e^{\rho(T-t)} \tilde{L}(z)^{\gamma}
$$

where $\rho$ is a positive constant s.t.

$$
\rho>\frac{\gamma}{1-\gamma} \frac{b^{2}+r^{2}+\sigma^{2} r(1-\gamma)}{\sigma^{2}}
$$

Proof. Fix $(t, z) \in[0, T] \times \overline{\mathcal{S}}$ and consider for some arbitrary $\alpha \in \mathcal{A}(t, z)$, the process $\tilde{L}\left(Z_{s}\right), t \leq s \leq T$, which is nonnegative by (4.4). By (4.9), we have :

$$
L\left(Z_{s}\right) \leq \tilde{L}\left(Z_{s}\right) \leq \tilde{L}\left(Z_{s}^{0, t, z}\right)=X_{s}^{0, t, x}+\frac{P_{s}^{0, t, p}}{\lambda}\left(1-e^{-\lambda y}\right), \quad t \leq s \leq T .
$$

From the arbitrariness of $\alpha$ and the nondecreasing property of $U$, we get the inequality in (4.18). From the growth condition (2.6) on the nonnegative function $U$ and since clearly $\left|X_{T}^{0, t, x}\right|^{\gamma}$ and $\left(P_{T}^{0, t, p}\right)^{\gamma}$ are integrable, i.e. in $L^{1}(\mathbb{P})$, we have $U\left(X_{T}^{0, t, x}+\frac{P_{T}^{0, t, p}}{\lambda}\left(1-e^{-\lambda y}\right)\right)$ $\in L^{1}(\mathbb{P})$. This clearly implies (4.19).

Consider now the nonnegative function :

$$
\varphi(t, z)=e^{\rho(T-t)} \tilde{L}(z)^{\gamma}=e^{\rho(T-t)}\left(x+\frac{p}{\lambda}\left(1-e^{-\lambda y}\right)\right)^{\gamma}
$$

and notice that $\varphi$ is smooth $C^{2}$ on $[0, T] \times\left(\overline{\mathcal{S}} \backslash D_{0}\right)$. Now, we claim that for $\rho$ large enough, the function $\varphi$ satisfies :

$$
-\frac{\partial \varphi}{\partial t}(t, z)-\mathcal{L} \varphi(t, z) \geq 0, \quad \forall(t, z) \in[0, T] \times \overline{\mathcal{S}} \backslash D_{0} .
$$

Indeed, a straightforward calculation shows that for all $t \in[0, T), z=(x, y, p) \in \overline{\mathcal{S}} \backslash D_{0}$ :

$$
\begin{aligned}
& -\frac{\partial \varphi}{\partial t}(t, z)-\mathcal{L} \varphi(t, z) \\
= & e^{\rho(T-t)} \tilde{L}(z)^{\gamma-2}\left[A x^{2}+B\left(\frac{p}{\lambda}\left(1-e^{-\lambda y}\right)\right)^{2}+2 C x \frac{p}{\lambda}\left(1-e^{-\lambda y}\right)\right],
\end{aligned}
$$

where

$$
A=\rho-r \gamma, \quad B=\rho-b \gamma+\frac{1}{2} \sigma^{2} \gamma(1-\gamma), \quad C=\rho-\frac{(b+r) \gamma}{2} .
$$

Hence, (4.23) is satisfied whenever $A>0$ and $B C-A^{2}>0$, which is the case for $\rho$ larger than the constant in the r.h.s. of (4.21).

Fix some $(t, z) \in[0, T) \times \overline{\mathcal{S}}$. If $z=(0,0, p)$ then we clearly have $v_{0}(t, z)=U(0)$ and so inequality (4.20) follows from $U(0) \leq K_{1}$ (see (2.6)). Consider now the case where $z \in$ $\overline{\mathcal{S}} \backslash D_{0}$ and notice that the process $Z_{s}^{0, t, z}=\left(X_{s}^{0, t, x}, y, P_{s}^{0, t, p}\right)$ never reaches $\{(0,0)\} \times \mathbb{R}_{+}^{*}$. Consider the stopping time

$$
T_{R}=\inf \left\{s \geq t:\left|Z_{s}^{0, t, z}\right|>R\right\} \wedge T
$$


so that the stopped process $\left(Z_{s \wedge T_{R}}^{0, t, z}\right)_{t \leq s \leq T}$ stays in the bounded set $\left\{z=(x, y, p) \in \overline{\mathcal{S}} \backslash D_{0}\right.$ : $|z| \leq R\}$ on which $\varphi(t,$.$) is smooth C^{2}$ and its derivative in $p, \frac{\partial \varphi}{\partial p}$ is bounded. By applying Itô's formula to $\varphi\left(s, Z_{s}^{0, t, z}\right)$ between $s=t$ and $s=T_{R}$, we have :

$$
\varphi\left(T_{R}, Z_{T_{R}}^{0, t, z}\right)=\varphi(t, z)+\int_{t}^{T_{R}}\left(\frac{\partial \varphi}{\partial t}+\mathcal{L} \varphi\right)\left(s, Z_{s}^{0, t, z}\right) d s+\int_{t}^{T_{R}} \frac{\partial \varphi}{\partial p}\left(s, Z_{s}^{0, t, z}\right) \sigma P_{s}^{0, t, p} d W_{s} .
$$

Since the integrand in the stochastic integral is bounded, we get by taking expectation in the last relation :

$$
\begin{aligned}
\mathbb{E}\left[\varphi\left(T_{R}, Z_{T_{R}}^{0, t, z}\right)\right] & =\varphi(t, z)+\mathbb{E}\left[\int_{t}^{T_{R}}\left(\frac{\partial \varphi}{\partial t}+\mathcal{L} \varphi\right)\left(s, Z_{s}^{0, t, z}\right) d s\right] \\
& \leq \varphi(t, z),
\end{aligned}
$$

where we used in the last inequality (4.23). Now, for almost $\omega \in \Omega$, for $R$ large enough ( $\geq$ $\bar{R}(\omega)$ ), we have $T_{R}=T$ so that $\varphi\left(T_{R}, Z_{T_{R}}\right)$ converges a.s. to $\varphi\left(T, Z_{T}\right)$. By Fatou's lemma, we deduce that $\mathbb{E}\left[\varphi\left(T, Z_{T}\right)\right] \leq \varphi(t, z)$. Since $\varphi(T, z)=\tilde{L}(z)^{\gamma}$, this yields

$$
\mathbb{E}\left[\tilde{L}\left(Z_{T}^{0, t, z}\right)^{\gamma}\right] \leq \varphi(t, z) .
$$

Finally, by the growth condition (2.6), this proves the required upper bound on the value function $v$.

Remark 4.2 The upper bound of the last proposition shows that the value function lies in the set of functions satisfying the growth condition :

$$
\mathcal{G}_{\gamma}([0, T] \times \overline{\mathcal{S}})=\left\{u:[0, T] \times \overline{\mathcal{S}} \longrightarrow \mathbb{R}, \sup _{[0, T] \times \overline{\mathcal{S}}} \frac{|u(t, z)|}{1+\left(x+\frac{p}{\lambda}\right)^{\gamma}}<\infty\right\} .
$$

Remark 4.3 The upper bound (4.19) is sharp in the sense that when $\lambda$ goes to zero (no price impact), we find the usual Merton bound :

$$
v(t, z) \leq \mathbb{E}\left[U\left(X_{T}^{0, t, x}+y P_{T}^{0, t, p}\right)\right] \leq K e^{\rho(T-t)}(x+p y)^{\gamma} .
$$

As a corollary, we can explicit the value function on the hyperplane of $\bar{S}$ :

$$
\bar{S}^{y}=\mathbb{R}_{+} \times\{0\} \times \mathbb{R}_{+}^{*} \subset \bar{S},
$$

where the agent does not hold any stock shares.

Corollary 4.5 For any $t \in[0, T), z=(x, 0, p) \in \bar{S}^{y}$, the investor optimally do not transact during $[t, T]$, i.e.

$$
v(t, z)=\mathbb{E}\left[U\left(X_{T}^{0, t, x}\right)\right]=U\left(x e^{r(T-t)}\right) .
$$

Proof. For $z=(x, 0, p) \in \bar{S}^{y}$, let us consider the no impulse control strategy starting from $z$ at $t$ which leads at the terminal date to a net wealth $L\left(Z_{T}^{0, t, z}\right)=X_{T}^{0, t, x}=x e^{r(T-t)}$. We then have $v(t, z) \geq \mathbb{E}\left[U\left(X_{T}^{0, t, x}\right)\right]=U\left(x e^{r(T-t)}\right)$. On the other hand, we have from (4.19): $v(t, z) \leq v_{0}(t, z)=\mathbb{E}\left[U\left(X_{T}^{0, t, x}\right)\right]$. This proves the required result. 


\subsection{Boundary properties}

We now turn to the behavior of the value function on the boundary of the solvency region. The situation is more complex than in models with proportional transaction costs where the boundary of the solvency region is an absorbing barrier and all transactions are stopped. Here, the behavior depends on which part of the boundary is the state, as showed in the following proposition.

Proposition 4.2 1) We have

$$
v=\mathcal{H} v \text { on }[0, T) \times\left(\partial_{\ell}^{-} \mathcal{S} \backslash D_{k} \cup \partial_{\ell}^{+} \mathcal{S}\right)
$$

and

$$
\mathcal{H} v=0 \text { on }[0, T) \times\left(\partial_{\ell}^{-} \mathcal{S} \cup \partial_{\ell}^{+, \lambda} \mathcal{S}\right) \cap \mathcal{N}_{\ell}
$$

2) We have

$$
v>\mathcal{H} v \text { on }[0, T) \times \partial^{y} \mathcal{S} \cup \partial_{0}^{x} \mathcal{S} \cup \partial_{1}^{x} \mathcal{S} \cup D_{k} .
$$

and

$$
\begin{aligned}
v & =0 \text { on }[0, T) \times D_{0}, \\
v(t, z) & =U\left(k e^{r(T-t)}\right), \quad(t, z) \in[0, T) \in D_{k} .
\end{aligned}
$$

Proof. 1. a) Fix some $(t, z) \in[0, T) \times\left(\partial_{\ell}^{-} \mathcal{S} \backslash D_{k} \cup \partial_{\ell}^{+} \mathcal{S}\right)$ and consider an arbitrary $\alpha=$ $\left(\tau_{n}, \zeta_{n}\right)_{n \geq 1} \in \mathcal{A}(t, z)$. We claim that $\tau_{1}=t$ a.s. i.e. one has to transact immediately at time $t$ in order to satisfy the solvency constraint.

$\star$ Consider first the case where $z \in \partial_{\ell}^{-} \mathcal{S} \backslash D_{k}$. Then on $\left[t, \tau_{1}\right], X_{s}=x e^{r(s-t)}, Y_{s}=y<$ $0, P_{s}=p P_{s}^{0}$, and so $L\left(Z_{s}\right)=L_{0}\left(Z_{s}^{0, t, z}\right)$. Hence, by integrating between $t$ and $\tau_{1}$, we get :

$$
0 \leq e^{-r\left(\tau_{1}-t\right)} L_{0}\left(Z_{\tau_{1}}^{0, t, z}\right)=\int_{t}^{\tau_{1}} e^{-r(u-t)} P_{u}^{0} y e^{-\lambda y}\left[(b-r) d u+\sigma d W_{u}\right]
$$

By Girsanov's theorem, one can define a probability measure $\mathbb{Q}$ equivalent to $\mathbb{P}$ under which $\hat{W}_{s}=W_{s}+(b-r) s / \sigma$ is a Brownian motion. Under this measure, the stochastic integral $\int_{t}^{\tau_{1}} e^{-r(u-t)} P_{u}^{0} y e^{-\lambda y} \sigma d \hat{W}_{u}$ has zero expectation from which we deduce with (4.31) that

$$
\int_{t}^{\tau_{1}} e^{-r(u-t)} P_{u}^{0} y e^{-\lambda y} \sigma d \hat{W}_{u}=0 \text { a.s. }
$$

Since $y \neq 0$ and $P_{s}^{0}>0$ a.s., this implies $\tau_{1}=t$ a.s.

$\star$ Consider the case where $z \in \partial_{\ell}^{+} \mathcal{S}$. Then on $\left[t, \tau_{1}\right], X_{s}=x e^{r(s-t)}<0, Y_{s}=y, P_{s}=$ $p P_{s}^{0}$, and so $L\left(Z_{s}\right)=L_{0}\left(Z_{s}\right)$. By the same argument as above, we deduce $\tau_{1}=t$.

By applying the dynamic programming principle (2.8) for $\tau=\tau_{1}$, we clearly deduce (4.26).

b) Fix some $(t, z=(x, y, p)) \in[0, T) \times\left(\partial_{\ell}^{-} \mathcal{S} \cup \partial_{\ell}^{+, \lambda} \mathcal{S}\right) \cap \mathcal{N}_{\ell}$. Then, from Lemma $4.2, \mathcal{C}(z)$ $=\{-y\}$ and so $\mathcal{H} v(t, z)=v(t, \Gamma(z,-y))=v(t, 0,0, p)$. Now, from Corollary 4.5, we have for all $z_{0}=(0,0, p) \in D_{0}, v\left(t, z_{0}\right)=U(0)=0$, which proves (4.29) and so (4.27). 
2. Fix some $t \in[0, T)$ and $z \in \partial^{y} \mathcal{S} \cup \partial_{0}^{x} \mathcal{S} \cup \partial_{1}^{x} \mathcal{S}$. Then by Lemma $4.2, \mathcal{C}(z)=\emptyset$, hence $\mathcal{H} v(t, z)=-\infty$ and so (4.28) is trivial. For $z=(k, 0, p) \in D_{k}$, we have by Lemma 4.2, $\mathcal{C}(z)$ $=\{0\}$ and so $\mathcal{H} v(t, z)=v(t, \Gamma(z, 0))=v(t, 0,0, p)=0$ by (4.29). Therefore, from Corollary 4.5 , we have for $z=(k, 0, p) \in D_{k}: v(t, z)=U\left(k e^{r(T-t)}\right)>0=\mathcal{H} v(t, z)$.

Remark 4.4 The last proposition and its proof means that when the state reaches $\partial_{\ell}^{-} \mathcal{S} \backslash$ $D_{k} \cup \partial_{\ell}^{+} \mathcal{S}$, one has to transact immediately since the no transaction strategy is not admissible. Moreover, if one is in $\left(\partial_{\ell}^{-} \mathcal{S} \cup \partial_{\ell}^{+, \lambda} \mathcal{S}\right) \cap \mathcal{N}_{\ell}$, one jumps directly to $D_{0}$ where all transactions are stopped. On the other hand, if the state is in $\partial^{y} \mathcal{S} \cup \partial_{0}^{x} \mathcal{S} \cup \partial_{1}^{x} \mathcal{S} \cup D_{k}$, one should do not transact : admissible transaction does not exist on $\partial^{y} \mathcal{S} \cup \partial_{0}^{x} \mathcal{S} \cup \partial_{1}^{x} \mathcal{S}$ while the only zero admissible transaction on $D_{k}$ is suboptimal with respect to the no transaction control. In the remaining part $\partial_{2}^{x} \mathcal{S}$ of the boundary, both decisions, transaction and no-transaction, are admissible : we only know that one of these decisions should be chosen optimally but we are not able to explicit which one is optimal. A summary of the behavior of the optimal strategy on the boundary of the solvency region is depicted in Figures 2 and 3.

The next result states the continuity of the value function on the part $D_{0}$ of the solvency boundary.

Corollary 4.6 The value function $v$ is continuous on $[0, T) \times D_{0}$ :

$$
\lim _{\left(t^{\prime}, z^{\prime}\right) \rightarrow(t, z)} v\left(t^{\prime}, z^{\prime}\right)=v(t, z)=0, \quad \forall(t, z) \in[0, T) \times D_{0} .
$$

Proof. This is a direct consequence of (4.19) and (4.29).

Remark 4.5 Notice that except on $D_{0}$, the value function is in general discontinuous on the boundary of the solvency region. More precisely, for any $t \in[0, T), z \in D_{k}$, we have from (4.26)-(4.27) :

$$
\lim _{\substack{z^{\prime} \rightarrow z \\ z^{\prime} \in \partial_{\ell}^{-} \mathcal{S} \backslash D_{k}}} v\left(t, z^{\prime}\right)=0
$$

while from Corollary 4.5 :

$$
\lim _{\substack{z^{\prime} \rightarrow z \\ z^{\prime} \in \bar{S}^{y}}} v\left(t, z^{\prime}\right)=U\left(k e^{r(T-t)}\right) .
$$

This shows that $v$ is discontinuous on $[0, T) \times D_{k}$. Similarly, one can show that $v$ is discontinuous on $[0, T) \times\left(\partial_{1}^{x} \mathcal{S} \cap \partial_{\ell}^{+} \mathcal{S}\right)$.

\subsection{Terminal condition}

We end this section by determining the terminal condition of the value function. As we shall see, this terminal condition takes into account the fact that just before the liquidation date $T$, one can do an impulse transaction : the effect is to lift-up the utility function $U_{L}$ through the impulse transaction operator $\mathcal{H}$. More precisely, we set

$$
v^{*}(T, z):=\limsup _{\substack{\left(t, z^{\prime}\right) \rightarrow(T, z) \\ t<T, z^{\prime} \in \mathcal{S}}} v\left(t, z^{\prime}\right), \quad v_{*}(T, z):=\liminf _{\substack{\left(t, z^{\prime}\right) \rightarrow(T, z) \\ t<T, z^{\prime} \in \mathcal{S}}} v\left(t, z^{\prime}\right)
$$


Proposition 4.3 We have

$$
v_{*}(T, z)=v^{*}(T, z)=\bar{U}(z), \quad \forall z \in \overline{\mathcal{S}}
$$

where

$$
\bar{U}(z):=\max \left[U_{L}(z), \mathcal{H} U_{L}(z)\right]
$$

Proof. 1) Fix some $z \in \overline{\mathcal{S}}$ and consider some sequence $\left(t_{m}, z_{m}\right)_{m} \in[0, T) \times \mathcal{S}$ converging to $(T, z)$ and s.t. $\lim _{m} v\left(t_{m}, z_{m}\right)=v_{*}(T, z)$. By taking the no impulse control strategy on $\left[t_{m}, T\right]$, we have

$$
v\left(t_{m}, z_{m}\right) \geq \mathbb{E}\left[U_{L}\left(Z_{T}^{0, t_{m}, z_{m}}\right)\right]
$$

Since $Z_{T}^{0, t_{m}, z_{m}}$ converges a.s. to $z$ when $m$ goes to infinity by continuity of the diffusion $Z^{0, t, z}$ in its initial conditions $(t, z)$, we deduce by Fatou's lemma that :

$$
v_{*}(T, z) \geq U_{L}(z) .
$$

Take now some arbitrary $\zeta \in \mathcal{C}(z)$. Consider first the case where $L(\Gamma(z, \zeta))>0$. We claim that for $m$ large enough, $\zeta \in \mathcal{C}\left(z_{m}\right)$. Indeed,

$\star$ suppose that $\zeta \neq-y$. Then, by continuity of the function $z^{\prime} \mapsto L\left(\Gamma\left(z^{\prime}, \zeta\right)\right)$ on $\left\{z^{\prime}=\right.$ $\left.\left(x^{\prime}, y^{\prime}, p^{\prime}\right): y^{\prime} \neq \zeta\right\}$, we deduce that $L\left(\Gamma\left(z_{m}, \zeta\right)\right)$ converges to $L(\Gamma(z, \zeta))>0$ and so for $m$ large enough, $\zeta \in \mathcal{C}\left(z_{m}\right)$.

$\star$ Suppose that $\zeta=-y$, i.e. $L(\Gamma(z, \zeta))=x+\ell(y, p)-k>0$. Notice that

$$
\begin{aligned}
L\left(\Gamma\left(z_{m}, \zeta\right)\right)=\max & {\left[L_{0}\left(z_{m}\right)-k+p g\left(-y, y_{m}\right), x_{m}+y e^{-\lambda y} p_{m}-k\right] 1_{y_{m}-y \geq 0} } \\
& +L_{0}\left(z_{m}\right) 1_{y_{m}-y<0}
\end{aligned}
$$

We then see that $\liminf _{m \rightarrow \infty} L\left(\Gamma\left(z_{m}, \zeta\right)\right) \geq L(\Gamma(z, \zeta))$, and so for $m$ large enough, $\zeta \in$ $\mathcal{C}\left(z_{m}\right)$

One may then consider the admissible control with immediate impulse at $t_{m}$ with size $\zeta$ and no other impulse until $T$ so that the associated state process is $Z^{t_{m}, z_{m}}=Z^{0, t_{m}, \Gamma\left(z_{m}, \zeta\right)}$ and thus

$$
v\left(t_{m}, z_{m}\right) \geq \mathbb{E}\left[U_{L}\left(Z_{T}^{0, t_{m}, \Gamma\left(z_{m}, \zeta\right)}\right)\right]
$$

Sending $m$ to infinity, we obtain :

$$
v_{*}(T, z) \geq U_{L}(\Gamma(z, \zeta))
$$

for all $\zeta$ in $\mathcal{C}(z)$ s.t. $L(\Gamma(z, \zeta))>0$. This last inequality (4.33) holds obviously true when $L(\Gamma(z, \zeta))=0$ since in this case $U_{L}(\Gamma(z, \zeta))=0 \leq v_{*}(T, z)$. By combining with (4.32), we get $v_{*}(T, z) \geq \bar{U}(z)$.

2) Fix some $z \in \overline{\mathcal{S}}$ and consider some sequence $\left(t_{m}, z_{m}\right)_{m} \in[0, T) \times \mathcal{S}$ converging to $(T, z)$ and s.t. $\lim _{m} v\left(t_{m}, z_{m}\right)=v^{*}(T, z)$. For any $m$, one can find $\hat{\alpha}^{m}=\left(\hat{\tau}_{n}^{m}, \hat{\zeta}_{n}^{m}\right)_{n} \in$ $\mathcal{A}\left(t_{m}, z_{m}\right)$ s.t.

$$
v\left(t_{m}, z_{m}\right) \leq \mathbb{E}\left[U_{L}\left(\hat{Z}_{T}^{m}\right)\right]+\frac{1}{m}
$$


where $\hat{Z}^{m}=\left(\hat{X}^{m}, \hat{Y}^{m}, \hat{P}^{m}\right)$ denotes the state process controlled by $\hat{\alpha}^{m}$ and given in $T$ by :

$$
\begin{aligned}
\hat{Z}_{T}^{m}=\hat{Z}_{T^{-}}^{m} & =z_{m}+\int_{t_{m}}^{T} B\left(\hat{Z}_{s}^{m}\right) d s+\int_{t_{m}}^{T} \Sigma\left(\hat{Z}_{s}^{m}\right) d W_{s}+\sum_{t_{m} \leq u<T} \Delta \hat{Z}_{s}^{m} \\
& =z_{m}+\left(\Gamma\left(z_{m}, \zeta_{1}^{m}\right)-z_{m}\right) 1_{\tau_{1}^{m}=t_{m}}+R_{T}^{m}
\end{aligned}
$$

with $B(z)=(r x, 0, b p)$ and $\Sigma(z)=(0,0, \sigma p)$ and

$$
R_{T}^{m}=\int_{t_{m}}^{T} B\left(\hat{Z}_{s}^{m}\right) d s+\int_{t_{m}}^{T} \Sigma\left(\hat{Z}_{s}^{m}\right) d W_{s}+\sum_{t_{m}<s<T} \Delta \hat{Z}_{s}^{m}
$$

We rewrite (4.34) as

$$
\begin{gathered}
v\left(t_{m}, z_{m}\right) \leq \mathbb{E}\left[\left\{U_{L}\left(\Gamma\left(z_{m}, \zeta_{1}^{m}\right)+R_{T}^{m}\right)-U_{L}\left(z_{m}+R_{T}^{m}\right)\right\} 1_{\tau_{1}^{m}=t_{m}}\right. \\
\left.+U_{L}\left(z_{m}+R_{T}^{m}\right)\right]+\frac{1}{m}
\end{gathered}
$$

We claim that $R_{T}^{m}$ converges a.s. to 0 as $m$ goes to infinity. Indeed, from the uniform bounds (4.11)-(4.12), we have

$$
\begin{aligned}
\left|B\left(\hat{Z}_{s}^{m}\right)\right|+\left|\Sigma\left(\hat{Z}_{s}^{m}\right)\right| & \leq(r+(b+\sigma) \lambda) \frac{e}{e-1} L\left(Z_{s}^{0, t, z_{m}}\right) \\
& \leq \operatorname{Cte} L\left(Z_{s}^{0, t, z}\right), \quad t_{m} \leq s \leq T, \text { a.s. }
\end{aligned}
$$

for some positive Cte independent of $m$. We then deduce that the Lebesgue and stochastic integral in (4.36) converge a.s. to zero as $m$ goes to infinity, i.e. $t_{m}$ goes to $T$. On the other hand, by same argument as in Remark 4.1, we see that for each $t_{m}<s<T$, the jump $\Delta Z_{s}^{m}$ is uniformly bounded in $m$. Moreover, by (4.17), we have

$$
N_{t_{m}}\left(\hat{\alpha}^{m}\right) \leq \frac{1}{k}\left[\bar{L}\left(\hat{Z}_{t_{m}}^{m}\right)-\bar{L}\left(\hat{Z}_{T^{-}}^{m}\right)+\int_{t_{m}}^{T}\left(r \hat{X}_{s}^{m}+\frac{\hat{P}_{s}^{m}}{\lambda}\right) d s+\int_{t_{m}}^{T} \frac{\sigma}{\lambda} \hat{P}_{s}^{m} d W_{s}\right]
$$

Similarly as above, by the uniform bounds in (4.11)-(4.12), the integrals in (4.38) converge to zero as $m$ goes to infinity. From the left-continuity of the state process $\hat{Z}^{m}$ and the continuity of $\bar{L}$, we deduce that $\bar{L}\left(\hat{Z}_{t^{m}}^{m}\right)-\bar{L}\left(Z_{T-}^{m}\right)$ converge to zero as $m$ goes to infinity. Therefore, $\sum_{t_{m}<s<T} \Delta \hat{Z}_{s}^{m}$ goes to zero as $m$ goes to infinity, which proves the required zero convergence of $R_{T}^{m}$.

By Remark 4.1, the sequence of jump size $\left(\zeta_{1}^{m}\right)_{m}$ is bounded, and up to a subsequence, converges, as $m$ goes to infinity, to some $\zeta \in \mathcal{C}(z)$. Moreover, it is easy to check that the family $\left\{U\left(X_{T}^{0, t_{m}, x_{m}}+\frac{P_{T}^{0, t_{m}, p_{m}}}{\lambda}\left(1-e^{-\lambda y_{m}}\right)\right), m \geq 1\right\}$ is uniformly integrable so that from (4.18), the family $\left\{U_{L}\left(\hat{Z}_{T}^{m}\right), m \geq 1\right\}$ is also uniformly integrable. Therefore, we can send $m$ to infinity into (4.34) (or (4.37)) by the dominated convergence theorem and get :

$$
\begin{aligned}
v^{*}(T, z) & \leq \mathbb{E}\left[\left\{U_{L}(\Gamma(z, \zeta))-U_{L}(z)\right\} \limsup _{m \rightarrow \infty} 1_{\tau_{1}^{m}=t_{m}}+U_{L}(z)\right] \\
& \leq \max \left\{U_{L}(z), \sup _{\zeta \in \mathcal{C}(z)} U_{L}(\Gamma(z, \zeta))\right\} .
\end{aligned}
$$

By completing with (4.32), this proves $v_{*}(T, z)=v^{*}(T, z)=\bar{U}(z)$. 
Remark 4.6 The previous result shows in particular that the value function is discontinuous on $T$. Indeed, recalling that we do not allow any impulse transaction at $T$, we have $v(T, z)=U_{L}(z)$ for all $z \in \overline{\mathcal{S}}$. Moreover, by Proposition 4.3, we have $v\left(T^{-}, z\right)=\bar{U}(z)$, hence $v(., z)$ is discontinuous on $T$ for all $z \in\left\{z \in \overline{\mathcal{S}}: \mathcal{H} U_{L}(z)>U_{L}(z)\right\} \neq \emptyset$.

\section{$5 \quad$ Viscosity characterization}

In this section, we intend to provide a rigorous characterization of the value function by means of (constrained) viscosity solution to the quasi-variational inequality :

$$
\min \left[-\frac{\partial v}{\partial t}-\mathcal{L} v, v-\mathcal{H} v\right]=0
$$

together with appropriate boundary conditions and terminal condition.

As mentioned previously, the value function is not known to be continuous a priori and so we shall work with the notion of discontinuous viscosity solutions. For a locally bounded function $u$ on $[0, T) \times \overline{\mathcal{S}}$ (which is the case of the value function $v$ ), we denote by $u_{*}$ (resp. $u^{*}$ ) the lower semi-continuous (lsc) (resp. upper semi-continuous (usc)) envelope of $u$. We recall that in general, $u_{*} \leq u \leq u^{*}$, and that $u$ is lsc iff $u=u_{*}, u$ is scs iff $u=u^{*}$, and $u$ is continuous iff $u_{*}=u^{*}(=u)$. We denote by $\operatorname{LSC}([0, T) \times \overline{\mathcal{S}})(\operatorname{resp} . \operatorname{USC}([0, T) \times \overline{\mathcal{S}}))$ the set of lsc (resp. usc) functions on $[0, T) \times \overline{\mathcal{S}}$.

We work with the suitable notion of constrained viscosity solutions, introduced in [26] for first-order equations, for taking into account boundary conditions arising in state constraints. The use of constrained viscosity solutions was initiated in [29] for stochastic control problems arising in optimal investment problems. The definition is given as follows :

Definition 5.1 (i) Let $\mathcal{O} \subset \overline{\mathcal{S}}$. A locally bounded function $u$ on $[0, T) \times \overline{\mathcal{S}}$ is a viscosity subsolution of $(5.1)$ in $[0, T) \times \mathcal{O}$ if for all $(\bar{t}, \bar{z}) \in[0, T) \times \mathcal{O}$ and $\varphi \in C^{1,2}([0, T) \times \overline{\mathcal{S}})$ s.t. $\left(u^{*}-\varphi\right)(\bar{t}, \bar{z})=0$ and $(\bar{t}, \bar{z})$ is a maximum of $u^{*}-\varphi$ on $[0, T) \times \mathcal{O}$, we have

$$
\min \left[-\frac{\partial \varphi}{\partial t}(\bar{t}, \bar{z})-\mathcal{L} \varphi(\bar{t}, \bar{z}), u^{*}(\bar{t}, \bar{z})-\mathcal{H} u^{*}(\bar{t}, \bar{z})\right] \leq 0 .
$$

(ii) Let $\mathcal{O} \subset \overline{\mathcal{S}}$. A locally bounded function $u$ on $[0, T) \times \overline{\mathcal{S}}$ is a viscosity supersolution of (5.1) in $[0, T) \times \mathcal{O}$ if for all $(\bar{t}, \bar{z}) \in[0, T) \times \mathcal{O}$ and $\varphi \in C^{1,2}([0, T) \times \overline{\mathcal{S}})$ s.t. $\left(u_{*}-\varphi\right)(\bar{t}, \bar{z})$ $=0$ and $(\bar{t}, \bar{z})$ is a minimum of $u_{*}-\varphi$ on $[0, T) \times \mathcal{O}$, we have

$$
\min \left[-\frac{\partial \varphi}{\partial t}(\bar{t}, \bar{z})-\mathcal{L} \varphi(\bar{t}, \bar{z}), u_{*}(\bar{t}, \bar{z})-\mathcal{H} u_{*}(\bar{t}, \bar{z})\right] \geq 0
$$

(iii) A locally bounded function $u$ on $[0, T) \times \overline{\mathcal{S}}$ is a constrained viscosity solution of (5.1) in $[0, T) \times \mathcal{S}$ if $u$ is a viscosity subsolution of $(5.1)$ in $[0, T) \times \overline{\mathcal{S}}$ and a viscosity supersolution of $(5.1)$ in $[0, T) \times \mathcal{S}$.

Remark 5.1 There is an equivalent formulation of viscosity solutions, which is useful for proving uniqueness results, see [10] : 
(i) Let $\mathcal{O} \subset \overline{\mathcal{S}}$. A function $u \in U S C([0, T) \times \overline{\mathcal{S}})$ is a viscosity subsolution of $(5.1)$ in $[0, T) \times \mathcal{O}$ if

$$
\min \left[-q_{0}-r x q_{1}-b p q_{3}-\frac{1}{2} \sigma^{2} p^{2} M_{33}, u(t, z)-\mathcal{H} u(t, z)\right] \leq 0,
$$

for all $(t, z=(x, y, p)) \in[0, T) \times \mathcal{O},\left(q_{0}, q=\left(q_{i}\right)_{1 \leq i \leq 3}, M=\left(M_{i j}\right)_{1 \leq i, j \leq 3}\right) \in \bar{J}^{2,+} u(t, z)$.

(ii) Let $\mathcal{O} \subset \overline{\mathcal{S}}$. A function $u \in L S C([0, T) \times \mathcal{S})$ is a viscosity supersolution of (5.1) in $[0, T) \times \mathcal{O}$ if

$$
\min \left[-q_{0}-r x q_{1}-b p q_{3}-\frac{1}{2} \sigma^{2} p^{2} M_{33}, u(t, z)-\mathcal{H} u(t, z)\right] \geq 0,
$$

for all $(t, z=(x, y, p)) \in[0, T) \times \mathcal{O},\left(q_{0}, q=\left(q_{i}\right)_{1 \leq i \leq 3}, M=\left(M_{i j}\right)_{1 \leq i, j \leq 3}\right) \in \bar{J}^{2,-} u(t, z)$.

(iii) A locally bounded function $u$ on $[0, T) \times \overline{\mathcal{S}}$ is a constrained viscosity solution to (5.1) if $u^{*}$ satisfies (5.4) for all $(t, z) \in[0, T) \times \overline{\mathcal{S}},(q, M) \in J^{2,+} u^{*}(t, z)$, and $u_{*}$ satisfies (5.5) for all $(t, z) \in[0, T) \times \mathcal{S},(q, M) \in J^{2,-} u_{*}(t, z)$.

Here $J^{2,+} u(t, z)$ is the parabolic second order superjet defined by :

$$
\begin{aligned}
& J^{2,+} u(t, z)=\left\{\left(q_{0}, q, M\right) \in \mathbb{R} \times \mathbb{R}^{3} \times \mathbb{S}^{3}:\right. \\
& \left.\lim _{\substack{\left(t^{\prime}, z^{\prime}\right) \rightarrow(t, z) \\
\left(t^{\prime}, z^{\prime}\right) \in[0, T) \times \mathcal{S}}} \frac{u\left(t^{\prime}, z^{\prime}\right)-u(t, z)-q_{0}\left(t^{\prime}-t\right)-q \cdot\left(z^{\prime}-z\right)-\frac{1}{2}\left(z^{\prime}-z\right) \cdot M\left(z^{\prime}-z\right)}{\left|t^{\prime}-t\right|+\left|z^{\prime}-z\right|^{2}} \leq 0\right\},
\end{aligned}
$$

$\mathbb{S}^{3}$ is the set of symmetric $3 \times 3$ matrices, $\bar{J}^{2,+} u(t, z)$ is its closure :

$$
\begin{gathered}
\bar{J}^{2,+} u(t, x)=\left\{\left(q_{0}, q, M\right)=\lim _{m \rightarrow \infty}\left(q_{0}^{m}, q^{m}, M^{m}\right) \quad \text { with }\left(q_{0}^{m}, q^{m}, M^{m}\right) \in J^{2,+} u\left(t_{m}, z_{m}\right)\right. \\
\text { and } \left.\lim _{m \rightarrow \infty}\left(t_{m}, z_{m}, u\left(t_{m}, z_{m}\right)\right)=(t, z, u(t, z))\right\},
\end{gathered}
$$

and $J^{2,-} u(t, x)=-J^{2,+}(-u)(t, x), \bar{J}^{2,-} u(t, x)=-\bar{J}^{2,+}(-u)(t, x)$.

\subsection{Viscosity property}

Our first main result of this section is the following.

Theorem 5.1 The value function $v$ is a constrained viscosity solution to $(5.1)$ in $[0, T) \times \mathcal{S}$.

Remark 5.2 The state constraint and the boundary conditions is translated through the PDE characterization via the subsolution property, which has to hold true on the whole closed region $\overline{\mathcal{S}}$. This formalizes the property that on the boundary of the solvency region, one of the two possible decisions, immediate impulse transaction or no-transaction, should be chosen optimally.

We need some auxiliary results on the impulse operator $\mathcal{H}$.

Lemma 5.1 Let $u$ be a locally bounded function on $[0, T) \times \overline{\mathcal{S}}$.

(i) $\mathcal{H} u_{*} \leq(\mathcal{H u})_{*}$. Moreover, if $u$ is lsc then $\mathcal{H} u$ is also lsc.

(ii) $\mathcal{H} u^{*}$ is usc and $(\mathcal{H u})^{*} \leq \mathcal{H} u^{*}$. 
Proof. (i) Let $\left(t_{n}, z_{n}\right)$ be a sequence in $[0, T) \times \overline{\mathcal{S}}$ converging to $(t, z)$ and s.t. $\mathcal{H} u\left(t_{n}, z_{n}\right)$ converges to $(\mathcal{H} u)_{*}(t, z)$. Then, using also the lowersemicontinuity of $u_{*}$ and the continuity of $\Gamma$, we have :

$$
\begin{aligned}
\mathcal{H} u_{*}(t, z) & =\sup _{\zeta \in \mathcal{C}(z)} u_{*}(t, \Gamma(z, \zeta)) \leq \sup _{\zeta \in \mathcal{C}(z)} \liminf _{n \rightarrow \infty} u_{*}\left(t_{n}, \Gamma\left(z_{n}, \zeta\right)\right) \\
& \leq \liminf _{n \rightarrow \infty} \sup _{\zeta \in \mathcal{C}(z)} u_{*}\left(t_{n}, \Gamma\left(z_{n}, \zeta\right)\right) \\
& \leq \lim _{n \rightarrow \infty} \mathcal{H} u\left(t_{n}, z_{n}\right)=(\mathcal{H} u)_{*}(t, z) .
\end{aligned}
$$

Suppose now that $u$ is lsc and let $(t, z) \in[0, T) \times \overline{\mathcal{S}}$ and let $\left(t_{n}, z_{n}\right)_{n>1}$ be a sequence in $[0, T) \times \overline{\mathcal{S}}$ converging to $(t, z)$ (as $n$ goes to infinity). By definition of the lsc envelope $(\mathcal{H} u)_{*}$, we then have :

$$
\mathcal{H} u(t, z)=\mathcal{H} u_{*}(t, z) \leq(\mathcal{H} u)_{*}(t, z) \leq \liminf _{n \rightarrow \infty} \mathcal{H} u\left(t_{n}, z_{n}\right),
$$

which shows the lower-semicontinuity of $\mathcal{H u}$.

(ii) Fix some $(t, z) \in[0, T) \times \overline{\mathcal{S}}$ and let $\left(t_{n}, z_{n}\right)_{n \geq 1}$ be a sequence in $[0, T) \times \overline{\mathcal{S}}$ converging to $(t, z)$ (as $n$ goes to infinity). Since $u^{*}$ is usc, $\Gamma$ is continuous, and $\mathcal{C}\left(z_{n}\right)$ is compact for each $n \geq 1$, there exists a sequence $\left(\hat{\zeta}_{n}\right)_{n \geq 1}$ with $\hat{\zeta}_{n} \in \mathcal{C}\left(z_{n}\right)$ such that :

$$
\mathcal{H} u^{*}\left(t_{n}, z_{n}\right)=u^{*}\left(t_{n}, \Gamma\left(z_{n}, \hat{\zeta}_{n}\right)\right), \quad \forall n \geq 1
$$

By Remark 4.1, the sequence $\left(\hat{\zeta}_{n}\right)_{n \geq 1}$ converges, up to a subsequence, to some $\hat{\zeta} \in \mathcal{C}(z)$. Therefore, we get :

$$
\mathcal{H} u^{*}(t, z) \geq u^{*}(t, \Gamma(z, \hat{\zeta})) \geq \limsup _{n \rightarrow \infty} u^{*}\left(t_{n}, \Gamma\left(z_{n}, \hat{\zeta}_{n}\right)\right)=\limsup _{n \rightarrow \infty} \mathcal{H} u^{*}\left(t_{n}, z_{n}\right),
$$

which proves that $\mathcal{H} u^{*}$ is usc.

On the other hand, fix some $(t, z) \in[0, T] \times \overline{\mathcal{S}}$ and let $\left(t_{n}, z_{n}\right)_{n \geq 1}$ be a sequence in $[0, T] \times \overline{\mathcal{S}}$ converging to $(t, z)$ and s.t. $\mathcal{H} u\left(t_{n}, z_{n}\right)$ converges to $(\mathcal{H} u)^{*}(t, z)$. Then, we have

$$
(\mathcal{H} u)^{*}(t, z)=\lim _{n \rightarrow \infty} \mathcal{H} u\left(t_{n}, z_{n}\right) \leq \limsup _{n \rightarrow \infty} \mathcal{H} u^{*}\left(t_{n}, z_{n}\right) \leq \mathcal{H} u^{*}(t, z),
$$

which shows that $(\mathcal{H} u)^{*} \leq \mathcal{H} u^{*}$.

We first prove the supersolution property.

Proof of supersolution property on $[0, T) \times \mathcal{S}$.

Let $(\bar{t}, \bar{z}) \in[0, T) \times \mathcal{S}$ and $\varphi \in C^{1,2}([0, T) \times \overline{\mathcal{S}})$ s.t. $v_{*}(\bar{t}, \bar{z})=\varphi(\bar{t}, \bar{z})$ and $\varphi \leq v_{*}$ on $[0, T) \times \mathcal{S}$. Recall that from (3.2), we have $v \geq \mathcal{H} v$ on $[0, T] \times \overline{\mathcal{S}}$. Combining with Lemma 5.1 (i), we obtain $\mathcal{H} v_{*}(\bar{t}, \bar{z}) \leq(\mathcal{H} v)_{*}(\bar{t}, \bar{z}) \leq v_{*}(\bar{t}, \bar{z})$, and so it remains to show that

$$
-\frac{\partial \varphi}{\partial t}(\bar{t}, \bar{z})-\mathcal{L} \varphi(\bar{t}, \bar{z}) \geq 0 .
$$

From the definition of $v_{*}$, there exists a sequence $\left(t_{m}, z_{m}\right)_{m \geq 1} \in[0, T) \times \mathcal{S}$ s.t. $\left(t_{m}, z_{m}\right)$ and $v\left(t_{m}, z_{m}\right)$ converge respectively to $(\bar{t}, \bar{z})$ and $v_{*}(\bar{t}, \bar{z})$ as $m$ goes to infinity. By continuity of $\varphi$, we also have that $\gamma_{m}:=v\left(t_{m}, z_{m}\right)-\varphi\left(t_{m}, z_{m}\right)$ converges to 0 as $m$ goes to infinity. 
Since $(\bar{t}, \bar{z}) \in[0, T) \times \mathcal{S}$, there exists $\eta>0$ s.t. for $m$ large enough, $t_{m}<T$ and $B\left(z_{m}, \eta / 2\right)$ $\subset B(\bar{z}, \eta):=\{|z-\bar{z}|<\eta\} \subset \mathcal{S}$. Let us then consider the admissible control in $\mathcal{A}\left(t_{m}, z_{m}\right)$ with no impulse until the first exit time $\tau_{m}$ before $T$ of the associated state process $Z_{s}=$ $Z_{s}^{0, t_{m}, z_{m}}$ from $B\left(z_{m}, \eta / 2\right)$ :

$$
\tau_{m}=\inf \left\{s \geq t_{m}:\left|Z_{s}^{0, t_{m}, z_{m}}-z_{m}\right| \geq \eta / 2\right\} \wedge T .
$$

Consider also a strictly positive sequence $\left(h_{m}\right)_{m}$ s.t. $h_{m}$ and $\gamma_{m} / h_{m}$ converge to zero as $m$ goes to infinity. By using the dynamic programming principle $(2.9)$ for $v\left(t_{m}, z_{m}\right)$ and $\hat{\tau}_{m}$ $:=\tau_{m} \wedge\left(t_{m}+h_{m}\right)$, we get :

$$
\begin{aligned}
v\left(t_{m}, z_{m}\right)=\gamma_{m}+\varphi\left(t_{m}, z_{m}\right) & \geq \mathbb{E}\left[v\left(\hat{\tau}_{m}, Z_{\hat{\tau}_{m}, t_{m}, z_{m}}^{0}\right)\right] \\
& \geq E\left[\varphi\left(\hat{\tau}_{m}, Z_{\hat{\tau}_{m}}^{0, t_{m}, z_{m}}\right),\right.
\end{aligned}
$$

since $\varphi \leq v_{*} \leq v$ on $[0, T) \times \mathcal{S}$. Now, by applying Itô's formula to $\varphi\left(s, Z_{s}^{0, t_{m}, z_{m}}\right)$ between $t_{m}$ and $\hat{\tau}_{m}$ and noting that the integrand of the stochastic integral term is bounded, we obtain by taking expectation :

$$
\frac{\gamma_{m}}{h_{m}}+\mathbb{E}\left[\frac{1}{h_{m}} \int_{t_{m}}^{\hat{\tau}_{m}}\left(-\frac{\partial \varphi}{\partial t}-\mathcal{L} \varphi\right)\left(s, Z_{s}^{0, t_{m}, z_{m}}\right) d s\right] \geq 0 .
$$

By continuity a.s. of $Z_{s}^{0, t_{m}, z_{m}}$, we have for $m$ large enough, $\hat{\tau}_{m}=t_{m}+h_{m}$, and so by the mean-value theorem, the random variable inside the expectation in (5.7) converges a.s. to $\left(-\frac{\partial \varphi}{\partial t}-\mathcal{L} \varphi\right)(\bar{t}, \bar{z})$ as $m$ goes to infinity. Since this random variable is also bounded by a constant independent of $m$, we conclude by the dominated convergence theorem and obtain (5.6).

We next prove the subsolution property.

Proof of subsolution property on $[0, T) \times \overline{\mathcal{S}}$.

Let $(\bar{t}, \bar{z}) \in[0, T) \times \overline{\mathcal{S}}$ and $\varphi \in C^{1,2}([0, T) \times \overline{\mathcal{S}})$ s.t. $v^{*}(\bar{t}, \bar{z})=\varphi(\bar{t}, \bar{z})$ and $\varphi \geq v^{*}$ on $[0, T) \times \overline{\mathcal{S}}$. If $v^{*}(\bar{t}, \bar{z}) \leq \mathcal{H} v^{*}(\bar{t}, \bar{z})$ then the subsolution inequality holds trivially. Consider now the case where $v^{*}(\bar{t}, \bar{z})>\mathcal{H} v^{*}(\bar{t}, \bar{z})$ and argue by contradiction by assuming on the contrary that

$$
\eta:=-\frac{\partial \varphi}{\partial t}(\bar{t}, \bar{z})-\mathcal{L} \varphi(\bar{t}, \bar{z})>0 .
$$

By continuity of $\varphi$ and its derivatives, there exists some $\delta_{0}>0$ s.t. $\bar{t}+\delta_{0}<T$ and for all $0<\delta \leq \delta_{0}$ :

$$
-\frac{\partial \varphi}{\partial t}(t, z)-\mathcal{L} \varphi(t, z)>\frac{\eta}{2}, \quad \forall(t, z) \in\left((\bar{t}-\delta)_{+}, \bar{t}+\delta\right) \times B(\bar{z}, \delta) \cap \overline{\mathcal{S}} .
$$

From the definition of $v^{*}$, there exists a sequence $\left(t_{m}, z_{m}\right)_{m \geq 1} \in\left((\bar{t}-\delta / 2)_{+}, \bar{t}+\delta / 2\right) \times$ $B(\bar{z}, \delta / 2) \cap \overline{\mathcal{S}}$ s.t. $\left(t_{m}, z_{m}\right)$ and $v\left(t_{m}, z_{m}\right)$ converge respectively to $(\bar{t}, \bar{z})$ and $v^{*}(\bar{t}, \bar{z})$ as $m$ goes to infinity. By continuity of $\varphi$, we also have that $\gamma_{m}:=v\left(t_{m}, z_{m}\right)-\varphi\left(t_{m}, z_{m}\right)$ converges to 0 as $m$ goes to infinity. By the dynamic programming principle (2.10), given $m \geq 1$, there exists $\hat{\alpha}^{m}=\left(\hat{\tau}_{n}^{m}, \hat{\zeta}_{n}^{m}\right)_{n \geq 1}$ s.t. for any stopping time $\tau$ valued in $\left[t_{m}, T\right]$, we have

$$
v\left(t_{m}, z_{m}\right) \leq \mathbb{E}\left[v\left(\tau, \hat{Z}_{\tau}^{m}\right)\right]+\frac{1}{m} .
$$


Here $\hat{Z}^{m}$ is the state process, starting from $z_{m}$ at $t_{m}$, and controlled by $\hat{\alpha}^{m}$. By choosing $\tau=\bar{\tau}^{m}:=\hat{\tau}_{1}^{m} \wedge \tau_{\delta}^{m}$ where

$$
\tau_{\delta}^{m}=\inf \left\{s \geq t_{m}: \hat{Z}_{s}^{m} \notin B\left(z_{m}, \delta / 2\right)\right\} \wedge\left(t_{m}+\delta / 2\right)
$$

is the first exit time before $t_{m}+\delta / 2$ of $\hat{Z}^{m}$ from the open ball $B\left(z_{m}, \delta / 2\right)$, we then get :

$$
\begin{aligned}
v\left(t_{m}, z_{m}\right) & \leq \mathbb{E}\left[v\left(\bar{\tau}^{m}, \hat{Z}_{\bar{\tau}^{m,-}}\right) 1_{\tau_{\delta}^{m}<\hat{\tau}_{1}^{m}}\right]+\mathbb{E}\left[v\left(\bar{\tau}^{m}, \Gamma\left(\hat{Z}_{\bar{\tau}^{m,-}}, \hat{\zeta}_{1}^{m}\right)\right) 1_{\hat{\tau}_{1}^{m} \leq \tau_{\delta}^{m}}\right]+\frac{1}{m} \\
& \leq \mathbb{E}\left[v\left(\bar{\tau}^{m}, \hat{Z}_{\bar{\tau}^{m,-}}\right) 1_{\tau_{\delta}^{m}<\hat{\tau}_{1}^{m}}\right]+\mathbb{E}\left[\mathcal{H} v\left(\bar{\tau}^{m}, \hat{Z}_{\bar{\tau}^{m,-}}\right) 1_{\hat{\tau}_{1}^{m} \leq \tau_{\delta}^{m}}\right]+\frac{1}{m} .
\end{aligned}
$$

Now, since $\mathcal{H} v \leq v \leq v^{*} \leq \varphi$ on $[0, T) \times \overline{\mathcal{S}}$, we obtain :

$$
\varphi\left(t_{m}, z_{m}\right)+\gamma_{m} \leq \mathbb{E}\left[\varphi\left(\bar{\tau}^{m}, \hat{Z}_{\bar{\tau}^{m,-}}\right)\right]+\frac{1}{m} .
$$

By applying Itô's formula to $\varphi\left(s, \hat{Z}_{s}^{m}\right)$ between $t_{m}$ and $\bar{\tau}_{m}$, we then get :

$$
\begin{aligned}
\gamma_{m} & \leq \mathbb{E}\left[\int_{t_{m}}^{\bar{\tau}_{m}}\left(\frac{\partial \varphi}{\partial t}+\mathcal{L} \varphi\right)\left(s, \hat{Z}_{s}^{m}\right) d s\right]+\frac{1}{m} \\
& \leq-\frac{\eta}{2} \mathbb{E}\left[\bar{\tau}_{m}-t_{m}\right]+\frac{1}{m}
\end{aligned}
$$

from (5.8). This implies

$$
\lim _{m \rightarrow \infty} \mathbb{E}\left[\bar{\tau}_{m}\right]=\bar{t}
$$

On the other hand, we have by (5.9)

$$
v\left(t_{m}, z_{m}\right) \leq \sup _{\substack{\left|t^{\prime}-t\right|<\delta \\\left|z^{\prime}-z\right|<\delta}} v\left(t^{\prime}, z^{\prime}\right) \mathbb{P}\left[\tau_{\delta}^{m}<\hat{\tau}_{1}^{m}\right]+\sup _{\substack{\left|t^{\prime}-t\right|<\delta \\\left|z^{\prime}-z\right|<\delta}} \mathcal{H} v\left(t^{\prime}, z^{\prime}\right) \mathbb{P}\left[\hat{\tau}_{1}^{m} \leq \tau_{\delta}^{m}\right]+\frac{1}{m}
$$

From (5.10), we then get by sending $m$ to infinity :

$$
v^{*}(\bar{t}, \bar{z}) \leq \sup _{\substack{\left|t^{\prime}-t\right|<\delta \\\left|z^{\prime}-z\right|<\delta}} \mathcal{H} v\left(t^{\prime}, z^{\prime}\right)
$$

Hence, sending $\delta$ to zero and by Lemma 5.1 (ii), we have

$$
\begin{aligned}
v^{*}(\bar{t}, \bar{z}) & \leq \lim _{\delta \downarrow 0} \sup _{\substack{\left|t^{\prime}-t\right|<\delta \\
\left|z^{\prime}-z\right|<\delta}} \mathcal{H} v\left(t^{\prime}, z^{\prime}\right)=(\mathcal{H} v)^{*}(\bar{t}, \bar{z}) \\
& \leq \mathcal{H}^{*} v(\bar{t}, \bar{z}),
\end{aligned}
$$

which is the required contradiction. 


\subsection{Comparison principle}

We finally turn to uniqueness question. Our next main result is a comparison principle for constrained (discontinuous) viscosity solutions to the quasi-variational inequality (5.1). It states that we can compare a viscosity subsolution to $(5.1)$ on $[0, T) \times \overline{\mathcal{S}}$ and a viscosity supersolution to $(5.1)$ on $[0, T) \times \mathcal{S}$, provided that we can compare them at the terminal date (as usual in parabolic problems) but also on the part $D_{0}$ of the solvency boundary.

Theorem 5.2 Suppose $u \in \mathcal{G}_{\gamma}([0, T] \times \overline{\mathcal{S}}) \cap U S C([0, T) \times \overline{\mathcal{S}})$ is a viscosity subsolution to $(5.1)$ in $[0, T) \times \overline{\mathcal{S}}$ and $w \in \mathcal{G}_{\gamma}([0, T] \times \overline{\mathcal{S}}) \cap L S C([0, T) \times \overline{\mathcal{S}})$ is a viscosity supersolution to $(5.1)$ in $[0, T) \times \mathcal{S}$ such that :

$$
\begin{aligned}
u(t, z) & \leq \liminf _{\left(t^{\prime}, z^{\prime}\right) \rightarrow(t, z)} w\left(t^{\prime}, z^{\prime}\right), \forall(t, z) \in[0, T) \times D_{0}, \\
u(T, z):=\lim _{\substack{\left(t, z^{\prime}\right) \rightarrow(T, z) \\
t<T, z^{\prime} \in \mathcal{S}}} u\left(t, z^{\prime}\right) & \leq w(T, z):=\liminf _{\substack{\left(t, z^{\prime}\right) \rightarrow(T, z) \\
t<T, z^{\prime} \in \mathcal{S}}} w\left(t, z^{\prime}\right), \quad \forall z \in \mathcal{S} .
\end{aligned}
$$

Then,

$$
u \leq w \text { on }[0, T] \times \mathcal{S}
$$

Remark 5.3 Notice that one cannot hope to derive a comparison principle in the whole closed region $\overline{\mathcal{S}}$ since it would imply the continuity of the value function on $\overline{\mathcal{S}}$, which is not true, see Remark 4.5.

In order to deal with the impulse obstacle in the comparison principle, we first produce some suitable perturbation of viscosity supersolutions. This strict viscosity supersolution argument was introduced by [18], and used e.g. in [1] for dealing with gradient constraints in singular control problem.

Lemma 5.2 Let $\gamma^{\prime} \in(0,1)$ and choose $\rho^{\prime}$ s.t.

$$
\rho^{\prime}>\frac{\gamma^{\prime}}{1-\gamma^{\prime}} \frac{b^{2}+r^{2}+\sigma^{2} r\left(1-\gamma^{\prime}\right)}{\sigma^{2}} \vee b \vee\left(\sigma^{2}-b\right)
$$

Given $\nu \geq 0$, consider the perturbation smooth function on $[0, T] \times \overline{\mathcal{S}}$ :

$$
\phi_{\nu}(t, z)=e^{\rho^{\prime}(T-t)}\left[\tilde{L}(z)^{\gamma^{\prime}}+\nu\left(\frac{e^{\lambda y}}{p}+p e^{-\lambda y}\right)\right] .
$$

Let $w \in L S C([0, T) \times \overline{\mathcal{S}})$ be a viscosity supersolution to $(5.1)$ in $[0, T) \times \mathcal{S}$. Then for any $m \geq 1$, any compact set $\mathcal{K}$ of $\mathbb{R} \times \mathbb{R} \times \mathbb{R}_{+}^{*}$, the usc function

$$
w_{m}=w+\frac{1}{m} \phi_{\nu}
$$

is a strict viscosity supersolution to (5.1) in $[0, T) \times \mathcal{S} \cap \mathcal{K}:$ there exists some constant $\delta$ (depending on $\mathcal{K}$ ) s.t.

$$
\min \left[-q_{0}-r x q_{1}-b p q_{3}-\frac{1}{2} \sigma^{2} p^{2} M_{33}, w_{m}(t, z)-\mathcal{H} w_{m}(t, z)\right] \geq \frac{\delta}{m},
$$


for all $(t, z=(x, y, p)) \in[0, T) \times \mathcal{O},\left(q_{0}, q=\left(q_{i}\right)_{1 \leq i \leq 3}, M=\left(M_{i j}\right)_{1 \leq i, j \leq 3}\right) \in \bar{J}^{2,-} w_{m}(t, z)$. Moreover, for $\gamma \in\left(0, \gamma^{\prime}\right)$ and $\nu>0$, if $w \in \mathcal{G}_{\gamma}([0, T] \times \overline{\mathcal{S}})$, and $u$ is also a function in $\mathcal{G}_{\gamma}([0, T] \times \overline{\mathcal{S}})$, then for any $t \in[0, T], m \geq 1$,

$$
\lim _{|z| \rightarrow \infty}\left(u-w_{m}\right)(t, z)=-\infty
$$

Proof. We set

$$
f_{1}(t, z)=e^{\rho^{\prime}(T-t)} \tilde{L}(z)^{\gamma^{\prime}}, \quad f_{2}(t, z)=e^{\rho^{\prime}(T-t)}\left(\frac{e^{\lambda y}}{p}+p e^{-\lambda y}\right) .
$$

From (4.5), we have for all $t \in[0, T), z \in \mathcal{S} \backslash \tilde{\mathcal{N}}=\{z \in \mathcal{S}: \tilde{L}(z) \geq k\}$ :

$$
f_{1}(t, \Gamma(z, \zeta)) \leq e^{\rho^{\prime}(T-t)}(\tilde{L}(z)-k)^{\gamma^{\prime}}, \quad \forall \zeta \in \mathcal{C}(z)
$$

and so

$$
\left(f_{1}-\mathcal{H} f_{1}\right)(t, z) \geq e^{\rho^{\prime}(T-t)}\left[\tilde{L}(z)^{\gamma^{\prime}}-(\tilde{L}(z)-k)^{\gamma^{\prime}}\right]>0
$$

Notice that relation (5.16) holds trivially true when $z \in \mathcal{N}$ since in this case $\mathcal{C}(z)=\emptyset$ and so $\mathcal{H} f(t, z)=-\infty$. We then deduce that for any compact set $\mathcal{K}$ of $\mathbb{R} \times \times \mathbb{R} \times \mathbb{R}_{+}^{*}$, there exists some constant $\delta_{0}>0$ s.t.

$$
f_{1}-\mathcal{H} f_{1} \geq \delta_{0}, \quad \text { on } \quad[0, T) \times \mathcal{S} \cap \mathcal{K} .
$$

Moreover, a direct calculation shows that for all $(t, z) \in[0, T] \times \overline{\mathcal{S}}, \zeta \in \mathcal{C}(z), f_{2}(t, \Gamma(z, \zeta))$ $=f_{2}(t, z)$, and so

$$
f_{2}-\mathcal{H} f_{2}=0
$$

This implies

$$
\begin{aligned}
\phi_{\nu}-\mathcal{H} \phi_{\nu} & =f_{1}+\nu f_{2}-\mathcal{H}\left(f_{1}+\nu f_{2}\right) \geq\left(f_{1}-\mathcal{H} f_{1}\right)+\nu\left(f_{2}-\mathcal{H} f_{2}\right) \\
& \geq \delta_{0}, \quad \text { on }[0, T) \times \mathcal{S} \cap \mathcal{K} .
\end{aligned}
$$

On the other hand, the same calculation as in (4.24) shows that for $\rho^{\prime}$ large enough, actually strictly larger than $\frac{\gamma^{\prime}}{1-\gamma^{\prime}} \frac{b^{2}+r^{2}+\sigma^{2} r\left(1-\gamma^{\prime}\right)}{\sigma^{2}}$, we have $-\frac{\partial f_{1}}{\partial t}-\mathcal{L} f_{1}>0$ on $[0, T) \times \mathcal{S}$. Hence, for any compact set $\mathcal{K}$ of $\mathbb{R} \times \times \mathbb{R} \times \mathbb{R}_{+}^{*}$, there exists some constant $\delta_{1}>0$ s.t.

$$
-\frac{\partial f_{1}}{\partial t}-\mathcal{L} f_{1} \geq \delta_{1} \quad \text { on } \quad[0, T) \times \mathcal{S} \cap \mathcal{K} .
$$

A direct calculation also shows that for all $(t, z) \in[0, T] \times \overline{\mathcal{S}}$ :

$$
\begin{aligned}
-\frac{\partial f_{2}}{\partial t}(t, z)-\mathcal{L} f_{2}(t, z) & =e^{\rho^{\prime}(T-t)}\left[\left(\rho^{\prime}+b-\sigma^{2}\right) \frac{e^{\lambda y}}{p}+\left(\rho^{\prime}-b\right) p e^{-\lambda y}\right] \\
& \geq 0,
\end{aligned}
$$


since $\rho^{\prime} \geq\left(\sigma^{2}-b\right) \vee b$. This implies that for any compact set $\mathcal{K}$ of $\mathbb{R} \times \times \mathbb{R} \times \mathbb{R}_{+}^{*}$, there exists some constant $\delta_{1}>0$ s.t.

$$
\begin{aligned}
-\frac{\partial \phi_{\nu}}{\partial t}-\mathcal{L} \phi_{\nu} & =-\frac{\partial f_{1}}{\partial t}-\mathcal{L} f_{1}+\nu\left(-\frac{\partial f_{2}}{\partial t}-\mathcal{L} f_{2}\right) \\
& \geq \delta_{1} \text { on }[0, T) \times \mathcal{S} \cap \mathcal{K} .
\end{aligned}
$$

By writing the viscosity supersolution property of $w$, we deduce from the inequalities (5.17)(5.18) the viscosity supersolution of $w_{m}$ to

$$
\min \left[-\frac{\partial w_{m}}{\partial t}-\mathcal{L} w_{m}, w_{m}-\mathcal{H} w_{m}\right] \geq \frac{\delta}{m} \quad \text { on } \quad[0, T) \times \mathcal{S} \cap \mathcal{K}
$$

and so (5.14), where we set $\delta=\delta_{0} \wedge \delta_{1}$. Finally, since $u, w \in \mathcal{G}_{\gamma}([0, T] \times \overline{\mathcal{S}})$, we have for some positive constant $K$ :

$$
\begin{aligned}
\left(u-w_{m}\right)(t, z) & \leq K\left[1+\left(x+\frac{p}{\lambda}\right)^{\gamma}\right]-\frac{1}{m}\left[\left(x+\frac{p}{\lambda}\right)^{\gamma^{\prime}}+\nu\left(\frac{e^{\lambda y}}{p}+p e^{-\lambda y}\right)\right] \\
& \longrightarrow-\infty, \quad \text { as }|z| \rightarrow \infty
\end{aligned}
$$

since $\gamma^{\prime}>\gamma$ and $\nu>0$.

We now follow general viscosity solution technique, based on the Ishii technique (see [10]) and adapt arguments from [17], [24] for handling with the nonlocal intervention operator $\mathcal{H}$ and [3], [1] for the boundary conditions.

\section{Proof of Theorem 5.2}

Let $u$ and $w$ as in Theorem 5.2. We (re)define $w$ on $[0, T) \times \partial \mathcal{S}$ by :

$$
w(t, z)=\liminf _{\substack{\left.\left(t^{\prime}, z^{\prime}\right) \rightarrow(t, z) \\\left(t^{\prime}, z^{\prime}\right) \in \overline{[0}, T\right) \times \mathcal{S}}} w\left(t^{\prime}, z^{\prime}\right), \quad \forall(t, z) \in[0, T) \times \partial \mathcal{S},
$$

and construct a strict viscosity supersolution to (5.1) according to Lemma 5.2, by considering for $m \geq 1$, the usc function on $[0, T) \times \overline{\mathcal{S}}$ :

$$
w_{m}=w+\frac{1}{m} \phi_{\nu}
$$

where $\phi_{\nu}$ is given in (5.13) for some given $\nu>0$. Recalling the definitions (5.12) of $u$ and $w$ on $\{T\} \times \overline{\mathcal{S}}$, we have then an extension of $u$ and $w_{m}$, which are usc and lsc on $[0, T] \times \overline{\mathcal{S}}$. In order to prove the comparison principle, it is sufficient to show that $\sup _{[0, T] \times \overline{\mathcal{S}}}\left(u-w_{m}\right)$ $\leq 0$ for all $m \geq 1$, since the required result is obtained by letting $m$ to infinity. We argue by contradiction and suppose that there exists some $m \geq 1$ s.t.

$$
\mu:=\sup _{[0, T] \times \overline{\mathcal{S}}}\left(u-w_{m}\right)>0 .
$$

Since $u-w_{m}$ is usc on $[0, T] \times \overline{\mathcal{S}}, \lim _{|z| \rightarrow \infty}\left(u-w_{m}\right)(z)=-\infty$ by $(5.15),\left(u-w_{m}\right)(T,$. 0 by $(5.12)$, and $\left(u-w_{m}\right)(t, z) \leq 0$ for $(t, z) \in[0, T) \times D_{0}$ by $(5.11)$, there exists a an open set $\mathcal{K}$ of $\mathbb{R} \times \mathbb{R} \times \mathbb{R}_{+}^{*}$ with closure $\overline{\mathcal{K}}$ compact s.t.

$$
\operatorname{Arg} \max _{[0, T] \times \overline{\mathcal{S}}}\left(u-w_{m}\right) \neq \emptyset \quad \subset \quad[0, T) \times \overline{\mathcal{S}} \backslash D_{0} \cap \mathcal{K} .
$$


Take then some $\left(t_{0}, z_{0}\right) \in[0, T) \times \overline{\mathcal{S}} \backslash D_{0} \cap \mathcal{K}$ s.t. $\mu=\left(u-w_{m}\right)\left(t_{0}, z_{0}\right)$ and distinguish the two cases :

- Case 1. : $z_{0} \in \partial \mathcal{S} \backslash D_{0} \cap \mathcal{K}$.

$\star$ From (5.19), there exists a sequence $\left(t_{i}, z_{i}\right)_{i \geq 1}$ in $[0, T) \times \mathcal{S} \cap \mathcal{K}$ converging to $\left(t_{0}, z_{0}\right)$ s.t. $w_{m}\left(t_{i}, z_{i}\right)$ tends to $w_{m}\left(t_{0}, z_{0}\right)$ when $i$ goes to infinity. We then set $\beta_{i}=\left|t_{i}-t_{0}\right|, \varepsilon_{i}=\left|z_{i}-z_{0}\right|$ and consider the function $\Phi_{i}$ defined on $[0, T]^{2} \times(\overline{\mathcal{S}} \cap \overline{\mathcal{K}})^{2}$ by :

$$
\begin{aligned}
\Phi_{i}\left(t, t^{\prime}, z, z^{\prime}\right)= & u(t, z)-w_{m}\left(t^{\prime}, z^{\prime}\right)-\varphi_{i}\left(t, t^{\prime}, z, z^{\prime}\right) \\
\varphi_{i}\left(t, t^{\prime}, z, z^{\prime}\right)= & \left|t-t_{0}\right|^{2}+\left|z-z_{0}\right|^{4} \\
& \quad+\frac{\left|t-t^{\prime}\right|^{2}}{2 \beta_{i}}+\frac{\left|z-z^{\prime}\right|^{2}}{2 \varepsilon_{i}}+\left(\frac{d\left(z^{\prime}\right)}{d\left(z_{i}\right)}-1\right)^{4} .
\end{aligned}
$$

Here $d(z)$ denotes the distance from $z$ to $\partial \mathcal{S}$. We claim that for $z_{0} \notin D_{0}$, there exists an open neighborhood $\mathcal{V}_{0} \subset \mathcal{K}$ of $z_{0}$ in which this distance function $d($.$) is twice continuously$ differentiable with bounded derivatives. This is well-known (see e.g. [15]) when $z_{0}$ lies in the smooth parts $\partial \mathcal{S} \backslash\left(D_{k} \cup C_{1} \cup C_{2}\right)$ of the boundary $\partial \mathcal{S}$. This is also true for $z_{0} \in$ $D_{k} \cup C_{1} \cup C_{2}$. Indeed, at these corner lines of the boundary, the inner normal vectors form an acute angle (positive scalar product) and thus one can extend from $z_{0}$ the boundary to a smooth boundary so that the distance $d$ is equal, locally on a neighborhood of $z_{0}$, to the distance to this smooth boundary. Notice that this is not true when $z_{0} \in D_{0}$, which forms a right angle. Now, since $\Phi_{i}$ is usc on the compact set $[0, T]^{2} \times(\overline{\mathcal{S}} \cap \overline{\mathcal{K}})^{2}$, there exists $\left(\hat{t}_{i}, \hat{t}_{i}^{\prime}, \hat{z}_{i}, \hat{z}_{i}^{\prime}\right) \in[0, T]^{2} \times(\overline{\mathcal{S}} \cap \overline{\mathcal{K}})^{2}$ that attains its maximum on $[0, T]^{2} \times(\overline{\mathcal{S}} \cap \overline{\mathcal{K}})^{2}:$

$$
\mu_{i}:=\sup _{[0, T]^{2} \times(\overline{\mathcal{S}} \cap \mathcal{K})^{2}} \Phi_{i}\left(t, t^{\prime}, z, z^{\prime}\right)=\Phi_{i}\left(\hat{t}_{i}, \hat{t}_{i}^{\prime}, \hat{z}_{i}, \hat{z}_{i}^{\prime}\right) .
$$

Moreover, there exists a subsequence, still denoted $\left(\hat{t}_{i}, \hat{t}_{i}^{\prime}, \hat{z}_{i}, \hat{z}_{i}^{\prime}\right)_{i \geq 1}$, converging to some $\left(\hat{t}_{0}, \hat{t}_{0}^{\prime}, \hat{z}_{0}, \hat{z}_{0}^{\prime}\right) \in[0, T]^{2} \times(\overline{\mathcal{S}} \cap \overline{\mathcal{K}})^{2}$. By writing that $\Phi_{i}\left(t_{0}, t_{i}, z_{0}, z_{i}\right) \leq \Phi_{i}\left(\hat{t}_{i}, \hat{t}_{i}^{\prime}, \hat{z}_{i}, \hat{z}_{i}^{\prime}\right)$, we have :

$$
\begin{aligned}
& u\left(t_{0}, z_{0}\right)-w_{m}\left(t_{i}, z_{i}\right)-\frac{1}{2}\left(\left|t_{i}-t_{0}\right|+\left|z_{i}-z_{0}\right|\right) \\
\leq & \mu_{i}=u\left(\hat{t}_{i}, \hat{z}_{i}\right)-w_{m}\left(\hat{t}_{i}^{\prime}, \hat{z}_{i}^{\prime}\right)-\left(\left|\hat{t}_{i}-t_{0}\right|^{2}+\left|\hat{z}_{i}-z_{0}\right|^{4}\right)-R_{i} \\
\leq & u\left(\hat{t}_{i}, \hat{z}_{i}\right)-w_{m}\left(\hat{t}_{i}^{\prime}, \hat{z}_{i}^{\prime}\right)-\left(\left|\hat{t}_{i}-t_{0}\right|^{2}+\left|\hat{z}_{i}-z_{0}\right|^{4}\right),
\end{aligned}
$$

where we set

$$
R_{i}=\frac{\left|\hat{t}_{i}-\hat{t}_{i}^{\prime}\right|^{2}}{2 \beta_{i}}+\frac{\left|\hat{z}_{i}-\hat{z}_{i}^{\prime}\right|^{2}}{2 \varepsilon_{i}}+\left(\frac{d\left(\hat{z}_{i}^{\prime}\right)}{d\left(z_{i}\right)}-1\right)^{4}
$$

From the boundedness of $u, w_{m}$ on $[0, T] \times \overline{\mathcal{S}} \cap \overline{\mathcal{K}}$, we deduce by inequality (5.23) the boundedness of the sequence $\left(R_{i}\right)_{i \geq 1}$, which implies $\hat{t}_{0}=\hat{t}_{0}^{\prime}$ and $\hat{z}_{0}=\hat{z}_{0}^{\prime}$. Then, by sending $i$ to infinity into (5.22) and (5.24), with the uppersemicontinuity (resp. lowersemicontinuity) of $u$ (resp. $\left.w_{m}\right)$, we obtain $\mu=u\left(t_{0}, z_{0}\right)-w_{m}\left(t_{0}, z_{0}\right) \leq u\left(\hat{t}_{0}, \hat{z}_{0}\right)-w_{m}\left(\hat{t}_{0}, \hat{z}_{0}\right)-\left|\hat{t}_{0}-t_{0}\right|^{2}$ $-\left|\hat{z}_{0}-z_{0}\right|^{4}$. By the definition of $\mu$, this shows :

$$
\hat{t}_{0}=\hat{t}_{0}^{\prime}=t_{0}, \quad \hat{z}_{0}=\hat{z}_{0}^{\prime}=z_{0}
$$


Sending again $i$ to infinity into (5.22)-(5.23)-(5.24), we thus derive that $\mu \leq \lim _{i} \mu_{i}=\mu-$ $\lim _{i} R_{i} \leq \mu$, and so

$$
\begin{array}{r}
\left.\mu_{i} \rightarrow \hat{t}_{i}^{\prime}\right|^{2} \\
2 \beta_{i}
\end{array}+\frac{\left|\hat{z}_{i}-\hat{z}_{i}^{\prime}\right|^{2}}{2 \varepsilon_{i}}+\left(\frac{d\left(\hat{z}_{i}^{\prime}\right)}{d\left(z_{i}\right)}-1\right)^{4} \rightarrow 0,
$$

as $i$ goes to infinity. In particular, for $i$ large enough, we have $\hat{t}_{i}, \hat{t}_{i}^{\prime}<T$ (since $t_{0}<T$ ), $d\left(\hat{z}_{i}^{\prime}\right) \geq d\left(z_{i}\right) / 2>0$, and so $\hat{z}_{i}^{\prime} \in \mathcal{S}$. For $i$ large enough, we may also assume that $\hat{z}_{i}, \hat{z}_{i}^{\prime}$ lie in the neighborhood $\mathcal{V}_{0}$ of $z_{0}$ so that the derivatives upon order 2 of $d$ at $\hat{z}_{i}$ and $\hat{z}_{i}^{\prime}$ exist and are bounded.

* We may then apply Ishii's lemma (see Theorem 8.3 in [10]) to $\left(\hat{t}_{i}, \hat{t}_{i}^{\prime}, \hat{z}_{i}, \hat{z}_{i}^{\prime}\right) \in[0, T) \times$ $[0, T) \times \overline{\mathcal{S}} \cap \mathcal{V}_{0} \times \mathcal{S} \cap \mathcal{V}_{0}$ that attains the maximum of $\Phi_{i}$ in (5.21). Hence, there exist $3 \times 3$ matrices $M=\left(M_{j l}\right)_{1 \leq j, l \leq 3}$ and $M^{\prime}=\left(M_{j l}^{\prime}\right)_{1 \leq j, l \leq 3}$ s.t. :

$$
\begin{aligned}
\left(q_{0}, q, M\right) & \in \bar{J}^{2,+} u\left(\hat{t}_{i}, \hat{z}_{i}\right), \\
\left(q_{0}^{\prime}, q^{\prime}, M^{\prime}\right) & \in \bar{J}^{2,-} w_{m}\left(\hat{t}_{i}^{\prime}, \hat{z}_{i}^{\prime}\right)
\end{aligned}
$$

where

$$
\begin{gathered}
q_{0}=\frac{\partial \varphi_{i}}{\partial t}\left(\hat{t}_{i}, \hat{t}_{i}^{\prime}, \hat{z}, \hat{t}_{i}^{\prime}\right), \quad q=\left(q_{j}\right)_{1 \leq j \leq 3}=D_{z} \varphi_{i}\left(\hat{t}_{i}, \hat{t}_{i}^{\prime}, \hat{z}_{i}, \hat{z}_{i}^{\prime}\right) \\
q_{0}^{\prime}=-\frac{\partial \varphi_{i}}{\partial t}\left(\hat{t}_{i}, \hat{t}_{i}^{\prime}, \hat{z}, \hat{t}_{i}^{\prime}\right), \quad q^{\prime}=\left(q_{j}^{\prime}\right)_{1 \leq j \leq 3}=-D_{z^{\prime}} \varphi_{i}\left(\hat{t}_{i}, \hat{t}_{i}^{\prime}, \hat{z}_{i}, \hat{z}_{i}^{\prime}\right) .
\end{gathered}
$$

and

$$
\left(\begin{array}{cc}
M & 0 \\
0 & -M^{\prime}
\end{array}\right) \leq D_{z, z^{\prime}}^{2} \varphi_{i}\left(\hat{t}_{i}, \hat{t}_{i}^{\prime}, \hat{z}_{i}, \hat{z}_{i}^{\prime}\right)+\varepsilon_{i}\left(D_{z, z^{\prime}}^{2} \varphi_{i}\left(\hat{t}_{i}, \hat{t}_{i}^{\prime}, \hat{z}_{i}, \hat{z}_{i}^{\prime}\right)\right)^{2}
$$

By writing the viscosity subsolution property (5.4) of $u$ and the strict viscosity supersolution (5.14) of $w_{m}$, we have :

$$
\begin{aligned}
\min \left[-q_{0}-r \hat{x}_{i} q_{1}-b \hat{p}_{i} q_{3}-\frac{1}{2} \sigma^{2} \hat{p}_{i}^{2} M_{33}, u\left(\hat{t}_{i}, \hat{z}_{i}\right)-\mathcal{H} u\left(\hat{t}_{i}, \hat{z}_{i}\right)\right] & \leq 0 \\
\min \left[-q_{0}^{\prime}-r \hat{x}_{i}^{\prime} q_{1}^{\prime}-b \hat{p}_{i}^{\prime} q_{3}^{\prime}-\frac{1}{2} \sigma^{2} \hat{p}_{i}^{\prime 2} M_{33}^{\prime}, w_{m}\left(\hat{t}_{i}^{\prime}, \hat{z}_{i}^{\prime}\right)-\mathcal{H} w_{m}\left(\hat{t}_{i}^{\prime}, \hat{z}_{i}^{\prime}\right)\right] & \geq \frac{\delta}{m} .
\end{aligned}
$$

We then distinguish the following two possibilities in (5.31) :

1. $u\left(\hat{t}_{i}, \hat{z}_{i}\right)-\mathcal{H} u\left(\hat{t}_{i}, \hat{z}_{i}\right) \leq 0$.

Since, from (5.32), we also have: $w_{m}\left(\hat{t}_{i}^{\prime}, \hat{z}_{i}^{\prime}\right)-\mathcal{H} w_{m}\left(\hat{t}_{i}^{\prime}, \hat{z}_{i}^{\prime}\right) \geq \frac{\delta}{m}$, we obtain by combining these two inequalities:

$$
\mu_{i} \leq u\left(\hat{t}_{i}, \hat{z}_{i}\right)-w_{m}\left(\hat{t}_{i}^{\prime}, \hat{z}_{i}^{\prime}\right) \leq \mathcal{H} u\left(\hat{t}_{i}, \hat{z}_{i}\right)-\mathcal{H} w_{m}\left(\hat{t}_{i}^{\prime}, \hat{z}_{i}^{\prime}\right)-\frac{\delta}{m}
$$

Sending $i$ to $\infty$, and by (5.26), we obtain :

$$
\begin{aligned}
\mu & \leq \limsup _{i \rightarrow \infty} \mathcal{H} u\left(\hat{t}_{i}, \hat{z}_{i}\right)-\liminf _{i \rightarrow \infty} \mathcal{H} w_{m}\left(\hat{t}_{i}^{\prime}, \hat{z}_{i}^{\prime}\right)-\frac{\delta}{m} \\
& \leq \mathcal{H} u\left(t_{0}, z_{0}\right)-\mathcal{H} w_{m}\left(t_{0}, z_{0}\right)-\frac{\delta}{m},
\end{aligned}
$$


from (5.25) and where we used the upper-semicontinuity of $\mathcal{H} u$ and the lower-semicontinuity of $\mathcal{H} w_{m}$ (see Lemma 5.1). By compactness of $\mathcal{C}\left(z_{0}\right)$, and since $u$ is usc, there exists some $\zeta_{0} \in \mathcal{C}\left(z_{0}\right)$ s.t. $\mathcal{H} u\left(t_{0}, z_{0}\right)=u\left(t_{0}, \Gamma\left(z_{0}, \zeta_{0}\right)\right)$. We then get the desired contradiction :

$$
\begin{aligned}
\mu & \leq \mathcal{H} u\left(t_{0}, z_{0}\right)-\mathcal{H} w_{m}\left(t_{0}, z_{0}\right)-\frac{\delta}{m} \\
& \leq u\left(t_{0}, \Gamma\left(z_{0}, \zeta_{0}\right)\right)-w_{m}\left(t_{0}, \Gamma\left(z_{0}, \zeta_{0}\right)\right)-\frac{\delta}{m} \leq \mu-\frac{\delta}{m} .
\end{aligned}
$$

2. $-q_{0}-r \hat{x}_{i} q_{1}-b \hat{p}_{i} q_{3}-\frac{1}{2} \sigma^{2} \hat{p}_{i}^{2} M_{33} \leq 0$.

Since, from (5.32), we also have: $-q_{0}^{\prime}-r \hat{x}_{i}^{\prime} q_{1}^{\prime}-b \hat{p}_{i}^{\prime} q_{3}^{\prime}-\frac{1}{2} \sigma^{2} \hat{p}_{i}^{\prime 2} M_{33}^{\prime} \geq \frac{\delta}{m}$, we obtain by combining these two inequalities :

$$
-\left(q_{0}-q_{0}^{\prime}\right)-r\left(\hat{x}_{i} q_{1}-\hat{x}_{i}^{\prime} q_{1}^{\prime}\right)-b\left(\hat{p}_{i} q_{3}-\hat{p}_{i}^{\prime} q_{3}^{\prime}\right)-\frac{1}{2} \sigma^{2}\left(\hat{p}_{i}^{2} M_{33}-\hat{p}_{i}^{\prime 2} M_{33}^{\prime}\right) \leq-\frac{\delta}{m} .
$$

Now, from (5.28)-(5.29), we explicit :

$$
\begin{aligned}
q_{0}=2\left(\hat{t}_{i}-t_{0}\right)+\frac{\left(\hat{t}_{i}-\hat{t}_{i}^{\prime}\right)}{\beta_{i}}, & q=4\left(\hat{z}_{i}-z_{0}\right)\left|\hat{z}_{i}-z_{0}\right|^{2}+\frac{\left(\hat{z}_{i}-\hat{z}_{i}^{\prime}\right)}{\varepsilon_{i}} \\
q_{0}^{\prime}=\frac{\left(\hat{t}_{i}-\hat{t}_{i}^{\prime}\right)}{\beta_{i}}, & q^{\prime}=\frac{\left(\hat{z}_{i}-\hat{z}_{i}^{\prime}\right)}{\varepsilon_{i}}-4 \operatorname{Dd}\left(\hat{z}_{i}^{\prime}\right)\left(\frac{d\left(\hat{z}_{i}^{\prime}\right)}{d\left(z_{i}\right)}-1\right)^{3}
\end{aligned}
$$

and we see by $(5.25)$ and $(5.27)$ that $q_{0}-q_{0}^{\prime}, \hat{x}_{i} q_{1}-\hat{x}_{i}^{\prime} q_{1}^{\prime}$ and $\hat{p}_{i} q_{3}-\hat{p}_{i}^{\prime} q_{3}^{\prime}$ converge to zero when $i$ goes to infinity. Moreover, from (5.30), we have :

$$
\frac{1}{2} \sigma^{2} \hat{p}_{i}^{2} M_{33}-\frac{1}{2} \sigma^{2} \hat{p}_{i}^{\prime 2} M_{33}^{\prime} \leq \mathcal{E}_{i}
$$

where

$$
\begin{aligned}
\mathcal{E}_{i} & =A_{i}\left(D_{z, z^{\prime}}^{2} \varphi_{i}\left(\hat{t}_{i}, \hat{t}_{i}^{\prime}, \hat{z}_{i}, \hat{z}_{i}^{\prime}\right)+\varepsilon_{i}\left(D_{z, z^{\prime}}^{2} \varphi_{i}\left(\hat{t}_{i}, \hat{t}_{i}^{\prime}, \hat{z}_{i}, \hat{z}_{i}^{\prime}\right)\right)^{2}\right) A_{i}^{\top} \\
& =A_{i}\left(\left(\begin{array}{cc}
\frac{1}{\varepsilon_{i}} I_{3}+P_{i} & -\frac{1}{\varepsilon_{i}} I_{3} \\
-\frac{1}{\varepsilon_{i}} I_{3} & \frac{1}{\varepsilon_{i}} I_{3}+Q_{i}
\end{array}\right)+\varepsilon_{i}\left(\begin{array}{cc}
\frac{1}{\varepsilon_{i}} I_{3}+P_{i} & -\frac{1}{\varepsilon_{i}} I_{3} \\
-\frac{1}{\varepsilon_{i}} I_{3} & \frac{1}{\varepsilon_{i}} I_{3}+Q_{i}
\end{array}\right)^{2}\right) A_{i}^{\top}
\end{aligned}
$$

with

$$
\begin{aligned}
A_{i} & =\left(0,0, \hat{p}_{i}, 0,0, \hat{p}_{i}^{\prime}\right) \\
P_{i} & =4\left|\hat{z}_{i}-z_{0}\right|^{2} I_{3}+8\left(\hat{z}_{i}-z_{0}\right)\left(\hat{z}_{i}-z_{0}\right)^{\top} \\
Q_{i} & =12\left(\frac{d\left(\hat{z}_{i}^{\prime}\right)}{d\left(\hat{z}_{i}^{\prime}\right)}-1\right)^{2} \operatorname{Dd}\left(\hat{z}_{i}^{\prime}\right) D d\left(\hat{z}_{i}^{\prime}\right)^{\top}+4\left(\frac{d\left(\hat{z}_{i}^{\prime}\right)}{d\left(\hat{z}_{i}^{\prime}\right)}-1\right)^{3} D^{2} d\left(\hat{z}_{i}^{\prime}\right) .
\end{aligned}
$$

Here $^{\top}$ denotes the transpose operator. After some straightforward calculation, we then get :

$$
\mathcal{E}_{i}=3 \frac{\left(\hat{p}_{i}^{\prime}-\hat{p}_{i}\right)^{2}}{\varepsilon_{i}}+A_{i}\left(\left(\begin{array}{cc}
3 P_{i} & -2 Q_{i} \\
-2 P_{i} & 3 Q_{i}
\end{array}\right)+\varepsilon_{i}\left(\begin{array}{cc}
P_{i}^{2} & 0 \\
0 & Q_{i}^{2}
\end{array}\right)\right) A_{i}^{\top},
$$


which converges also to zero from (5.25) and (5.27). Therefore, by sending $i$ to infinity into (5.33), we see that the limsup of its l.h.s. is nonnegative, which gives the required contradiction : $0 \leq-\delta / m$.

- Case 2. : $z_{0} \in \mathcal{S} \cap \mathcal{K}$.

This case is dealt similarly as in Case 1 . and its proof is omitted. It suffices e.g. to consider the function

$$
\begin{aligned}
& \Psi_{i}\left(t, z, z^{\prime}\right)=u(t, z)-w_{m}\left(t, z^{\prime}\right)-\psi_{i}\left(t, z, z^{\prime}\right) \\
& \psi_{i}\left(t, z, z^{\prime}\right)=\left|t-t_{0}\right|^{2}+\left|z-z_{0}\right|^{4}+\frac{i}{2}\left|z-z^{\prime}\right|^{2}
\end{aligned}
$$

for $i \geq 1$, and to take a maximum $\left(\tilde{t}_{i}, \tilde{z}_{i}, \tilde{z}_{i}^{\prime}\right)$ of $\Psi_{i}$. We then show that the sequence $\left(\tilde{t}_{i}, \tilde{z}_{i}, \tilde{z}_{i}^{\prime}\right)_{i \geq 1}$ converges to $\left(t_{0}, z_{0}, z_{0}\right)$ as $i$ goes to infinity and we apply Ishii's lemma to get the required contradiction.

By combining previous results, we then finally obtain the following PDE characterization of the value function.

Corollary 5.1 The value function $v$ is continuous on $[0, T) \times \mathcal{S}$ and is the unique (in $[0, T) \times \mathcal{S})$ constrained viscosity solution to $(5.1)$ lying in $\mathcal{G}_{\gamma}([0, T] \times \overline{\mathcal{S}})$ and satisfying the boundary condition :

$$
\lim _{\left(t^{\prime}, z^{\prime}\right) \rightarrow(t, z)} v\left(t^{\prime}, z^{\prime}\right)=0, \quad \forall(t, z) \in[0, T) \times D_{0},
$$

and the terminal condition

$$
v\left(T^{-}, z\right):=\lim _{\substack{\left(t, z^{\prime}\right) \rightarrow(T, z) \\ t<T, z^{\prime} \in \mathcal{S}}} v\left(t, z^{\prime}\right)=\bar{U}(z), \quad \forall z \in \overline{\mathcal{S}}
$$

Proof. From Theorem 5.1, $v^{*}$ is an usc viscosity subsolution to $(5.1)$ in $[0, T) \times \overline{\mathcal{S}}$ and $v_{*}$ is a lsc viscosity supersolution to $(5.1)$ in $[0, T) \times \mathcal{S}$. Moreover, by Corollary 4.6 and Proposition 4.3, we have $v^{*}(t, z)=v_{*}(t, z)=0$ for all $(t, z) \in[0, T) \times D_{0}$, and $v^{*}(T, z)=$ $v_{*}(T, z)=\bar{U}(z)$ for all $z \in \overline{\mathcal{S}}$. Then by Theorem 5.2 , we deduce $v^{*} \leq v_{*}$ on $[0, T] \times \mathcal{S}$, which proves the continuity of $v$ on $[0, T) \times \mathcal{S}$. On the other hand, suppose that $\tilde{v}$ is another constrained viscosity solution to (5.1) with $\lim _{\left(t^{\prime}, z^{\prime}\right) \rightarrow(t, z)} v\left(t^{\prime}, z^{\prime}\right)=0$ for $(t, z) \in[0, T) \times D_{0}$ and $\tilde{v}\left(T^{-}, z\right)=\bar{U}(z)$ for $z \in \overline{\mathcal{S}}$. Then, $\tilde{v}^{*}(t, z)=v_{*}(t, z)=v^{*}(t, z)=\tilde{v}_{*}(t, z)$ for $(t, z) \in$ $[0, T) \times D_{0}$ and $\tilde{v}^{*}(T, z)=v_{*}(T, z)=v^{*}(T, z)=\tilde{v}_{*}(T, z)$ for $z$ in $\overline{\mathcal{S}}$. We then deduce by Theorem 5.2 that $v^{*} \leq \tilde{v}_{*} \leq \tilde{v}^{*} \leq v_{*}$ on $[0, T] \times \mathcal{S}$. This proves $v=\tilde{v}$ on $[0, T) \times \mathcal{S}$.

\section{Conclusion}

We formulated a model for optimal portfolio selection under liquidity risk and price impact. Our main result is a characterization of the value function as the unique constrained viscosity solution to the quasi-variational Hamilton-Jacobi-Bellman inequality associated to this impulse control problem under solvency constraints. The main technical difficulties come from the nonlinearity due to price impact, and the state constraint. They are overcomed 
with the specific exponential form of the price impact function : a natural theoretical question is to extend our results for general price impact functions. Once we have provided a complete PDE characterization of the value function, the next step, from an applied view point, is to numerically solve this quasi-variational inequality. This can be realized for instance by iterated optimal stopping problems as done in [9]. Moreover, from an economic viewpoint, it would be of course interesting to analyse the effects of liquidity risk and price impact in our model on the optimal portfolio in a classical market without frictions, e.g. the Merton model. These numerical and economical studies are the topics of a forthcoming work, see [23].

\section{References}

[1] Akian M., Sulem A. and M. Taksar (2001) : "Dynamic optimization of long term growth rate for a portfolio with transaction costs and logarithm utility", Math. Finance, 11, 153-188.

[2] Back K. (1992) : "Insider trading in continuous time", Review of Financial Studies, 5, 387-409.

[3] Barles G. (1994) : Solutions de viscosité des équations d'Hamilton-Jacobi, Math. et Appli., Springer Verlag.

[4] Bensoussan A. and J.L. Lions (1982) : Impulse control and quasi-variational inequalities, Gauthiers-Villars.

[5] Bank P. and D. Baum (2004) : "Hedging and portfolio optimization in illiquid financial markets with a large trader", Mathematical Finance, 14, 1-18.

[6] Cadenillas A. and F. Zapatero (1999) : "Optimal central bank intervention in the foreign exchange market", Journal of Econ. Theory, 97, 218-242.

[7] Cetin U., Jarrow R. and P. Protter (2004) : "Liquidity risk and arbitrage pricing theory", Finance and Stochastics, 8, 311-341.

[8] Cetin U. and C. Rogers (2005) : "Modelling liquidity effects in discrete-time", to appear in Mathematical Finance.

[9] Chancelier J.P., Oksendal B. and A. Sulem (2001) : "Combined stochastic control and optimal stopping, and application to numerical approximation of combined stochastic and impulse control", Stochastic Financial Mathematics, Proc. Steklov Math. Inst. Moscou, 149-175, ed. A. Shiryaev.

[10] Crandall M., Ishii H. and P.L. Lions (1992) : "User's guide to viscosity solutions of second order partial differential equations", Bull. Amer. Math. Soc., 27, 1-67.

[11] Cuoco D. and J. Cvitanic (1998) : "Optimal consumption choice for a large investor", Journal of Economic Dynamics and Control, 22, 401-436.

[12] Davis M. and A. Norman (1990) : "Portfolio selection with transaction costs", Math. of Oper. Research, 15, 676-713.

[13] Fleming W. and M. Soner (1993) : Controlled Markov processes and viscosity solutions, Springer Verlag, New York.

[14] Frey R. (1998) : "Perfect option hedging for a large trader", Finance and Stochastics, 2, 115-141. 
[15] Gilbarg D. and N. Trudinger (1977) : Elliptic partial differential equations of second order, Springer Verlag, Berlin.

[16] He H. and H. Mamaysky (2005) : "Dynamic trading policies with price impact", Journal of Economic Dynamics and Control, 29, 891-930.

[17] Ishii K. (1993) : "Viscosity solutions of nonlinear second order elliptic PDEs associated with impluse control problems", Funkcial. Ekvac., 36, 123-141.

[18] Ishii H. and P.L. Lions (1990) : "Viscosity solutions of fully nonlinear second order elliptic partial differential equations", Journal of Differential equations, 83, 26-78.

[19] Jeanblanc M. and A. Shiryaev (1995) : "Optimization of the flow of dividends", Russian Math Surveys, 50, 257-277.

[20] Jouini E. and H. Kallal (1995) : "Martingale and arbitrage in securities markets with transaction costs", Journal of Econ. Theory, 66, 178-197.

[21] Korn R. (1998) : "Portfolio optimization with strictly positive transaction costs and impulse control", Finance and Stochastics, 2, 85-114.

[22] Kyle A. (1985) : "Continuous auctions and insider trading", Econometrica, 53, 1315-1335.

[23] Ly Vath V., Mnif M. and H. Pham (2005) : "Numerical approximation for an impulse control problem arising in portfolio selection under liquidity risk", in preparation.

[24] Oksendal B. and A. Sulem (2002) : "Optimal consumption and portfolio with both fixed and proportional transaction costs", SIAM J. Cont. Optim., 40, 1765-1790.

[25] Papanicolaou G. and R. Sircar (1998) : "General Black-Scholes models accounting for increased market volatility from hedging strategies", Applied Mathematical Finance, 5, 45-82.

[26] Soner H. (1986) : "Optimal control with state-space constraint, I and II", SIAM J. Cont. Optim., 24, 552-561, and 1110-1122.

[27] Subramanian A. and R. Jarrow (2001) : "The liquidity discount", Mathematical Finance, 11, 447-474.

[28] Tang S. and J. Yong (1993) : "Finite horizon stochastic optimal switching and impulse controls with a viscosity solution approach", Stoch. and Stoch. Reports, 45, 145-176.

[29] Zariphopoulou T. (1988) : Optimal investment-consumption models with constraints, Phd Thesis, Brown University. 